\title{
A Wavelet Theory for Local Fields and Related Groups
}

\author{
By John J. Benedetto and Robert L. Benedetto
}

\begin{abstract}
Let $G$ be a locally compact abelian group with compact open subgroup $H$. The best known example of such a group is $G=\mathbb{Q}_{p}$, the field of p-adic rational numbers (as a group under addition), which has compact open subgroup $H=\mathbb{Z}_{p}$, the ring of p-adic integers. Classical wavelet theories, which require a non trivial discrete subgroup for translations, do not apply to $G$, which may not have such a subgroup. A wavelet theory is developed on $G$ using coset representatives of the discrete quotient $\widehat{G} / H^{\perp}$ to circumvent this limitation. Wavelet bases are constructed by means of an iterative method giving rise to so-called wavelet sets in the dual group $\widehat{G}$. Although the Haar and Shannon wavelets are naturally antipodal in the Euclidean setting, it is observed that their analogues for $G$ are equivalent.
\end{abstract}

\section{Introduction}

\subsection{Background}

Every locally compact abelian group (LCAG) $G$ is topologically and algebraically isomorphic to $\mathbb{R}^{d} \times G_{o}$, where $\mathbb{R}^{d}$ is Euclidean space and $G_{o}$ is a LCAG containing a compact open subgroup $H_{o}$; note that a subgroup $H$ of $G$ is open if and only if $G / H$ is discrete. Because of applications, e.g., in signal processing, and because of the role of periodization, most wavelet theory has been constructed on $\mathbb{R}^{d}$ or on LCAGs containing cocompact discrete subgroups, e.g., see [46, 23, 17, 43, 24, 45] for the multiresolution analysis (MRA) theory, see $[59,19,20,30,56,10,11,12]$ for the theory of wavelet sets, and see, $[48,5,6,16,47]$ for unifying general approaches. There has also been work on wavelet theory outside of the Euclidean setting, e.g., [42, 18, 33, 58, 2, 34, 4, 29, 54, 1, 3], and classical work on harmonic analysis on local fields, e.g., [57] and [22]. In this article, we shall deal with wavelet theory for functions defined on groups $G_{o}$. Our results are outlined in Section 1.2.

We are motivated by our interest in nondiscrete locally compact fields such as finite extensions of the $p$-adic rationals $\mathbb{Q}_{p}$. We also wish to lay some of the groundwork for a wavelet theory in the adelic setting, with the goal of establishing another technique from harmonic analysis for

\footnotetext{
Math Subject Classifications. Primary: 42C40; secondary: 11S99, 42C15, 43A70.

Key Words and Phrases. Wavelet, locally compact abelian group, p-adic field.

Acknowledgements and Notes. The first author gratefully acknowledges support from NSF DMS Grant 0139759

and ONR Grant N000140210398; the second author gratefully acknowledges support from NSF DMS Grant 0071541.
}

(1) 2004 The Journal of Geometric Analysis ISSN 1050-6926 
certain number theoretic problems formulated in terms of adeles. To these ends, this article is devoted to the construction of a wavelet theory on local fields.

\subsection{Results}

Let $G$ be a LCAG with compact open subgroup $H \subseteq G$. Besides the cases that $G$ is compact or discrete, there are many standard interesting examples of groups $G$ with compact open subgroups; see Section 2.3 below, as well as [14]. We develop a constructive theory of wavelets in $L^{2}(G)$ by means of the theory of wavelet sets mentioned in Section 1.1. In fact, we formulate a computable algorithm for generating wavelet orthonormal bases (ONBs) for $L^{2}(G)$ depending on given expansive automorphisms $A$ and given easily constructible mappings $T$. We envisage that $A$ and $T$ will be chosen so that the resulting wavelet ONB will have properties desirable for a given problem.

The algorithm is stated in Section 4.1. The proof that the algorithm does in fact generate wavelet ONBs is given by Theorem 4.2 in Section 4.2. Theorem 4.2 depends on Theorem 3.5 in Section 3.2. Theorem 3.5 is geometrical in nature; and it proves the equivalence of the existence of wavelets in terms of sets $\Omega$ that are both "translation" and "dilation" tiles for the dual group $\widehat{G}$.

The geometry of $G$ is decidedly non Euclidean, and $G$ may not contain a suitable discrete subgroup to serve as a lattice for translation. To circumvent this problem we introduce the idea of $(\tau, \mathcal{D})$-congruence in Sections 2.1 and 3.1. Furthermore, a notion of expansive automorphism is required to define dilation properly. This notion and its properties are formulated and proved in Section 2.2. Section 2.3 is devoted to number theoretic examples and their behavior under our new "translation" and "dilation" operations.

Finally, Section 5 provides examples of wavelet ONBs for $L^{2}(G)$ generated by our algorithm. In particular, we prove in Proposition 5.1 that the analogues of the Euclidean Haar and Shannon wavelets are one and the same over $G$. This section also gives us an opportunity to compare our method and its generality with results of Lang [39, 40,41], which preceded this work, and Kozyrev [36], which was brought to our attention after our main results were proved, announced, and written in a preliminary version of this article. Because of the more restrictive goals of Lang and Kozyrev, our results are significantly more general than theirs, and our methods are fundamentally different.

Since we have to use the notion of an expansive automorphism, we hasten to reaffirm that our method is not classical MRA reformulated for $G$. Our MRA theory of $L^{2}(G)$ is presented in [9].

For all of its effectiveness in the Euclidean setting, MRA wavelet ONBs require several steps of verification (e.g., using expansive linear transformations to prove the density of $\bigcup V_{j}$ for an MRA $\left.\left\{V_{j}\right\}\right)$ not necessary in our algorithm. On the other hand, the inherent complexity of a wavelet set $\Omega$ and the slow wavelet decay of our wavelets $\check{\mathbf{1}}_{\Omega}$ on $\mathbb{R}^{d}$ are not a major issue for $G$, since $\mathbf{1}_{H^{\perp}}$ is locally constant, that is, every point $\gamma \in \widehat{G}$ has a neighborhood $U$ on which $\mathbf{1}_{H^{\perp}}$ is constant. This is true because $H^{\perp}$ is both an open and a closed subset of $\widehat{G}$. ( $\check{\mathbf{1}}_{\Omega}$ is the inverse Fourier transform of the characteristic function $\mathbf{1}_{\Omega}$ of $\Omega$ and $H^{\perp}$ is the annihilator of $H$; see Section 1.3.) Thus, $\mathbf{1}_{H^{\perp}}$ is locally smooth (meaning that $\mathbf{1}_{H^{\perp}}$ would be " $C^{\infty}$ " when there is a suitable notion of derivative of a map from $\widehat{G}$ to $\mathbb{C}$ ) with compact support. Its inverse Fourier transform is $\mathbf{1}_{H}$, which is also locally constant and hence is also " $C^{\infty}$ " with compact support. Also, for a large class of the constructible mappings $T$, the approximants to $\mathbf{1}_{\Omega}$ at any finite step of our algorithm are locally constant with compact support, as are the inverse transforms of such approximants. This inverse Fourier transform generates a wavelet frame; see [13] for the 
Euclidean case. Of course, the limit $\mathbf{1}_{\Omega}$ itself is not necessarily locally constant, although it does have compact support.

\subsection{Prerequisites and notation}

Let $G$ be a LCAG, and denote its dual group by $\widehat{G}$. Let $H$ be a closed subgroup of $G$.

$$
H^{\perp}=\{\gamma \in \widehat{G}: \forall x \in H, \quad(x, \gamma)=1\} \subseteq \widehat{G}
$$

is the annihilator subgroup of $H$ in $\widehat{G}$, where $(x, \gamma)$ denotes the action of the duality between $G$ and $\widehat{G}$.

$\widehat{G / H}$ is algebraically and topologically isomorphic to $H^{\perp}$, and $\widehat{H}$ is algebraically and topologically isomorphic to $\widehat{G} / H^{\perp} . H^{\perp}$ is compact if and only if $H$ is open in $G$; and $H$ is compact if and only if $H^{\perp}$ is open in $\widehat{G}$. We shall suppress "algebraically and topologically isomorphic" and write, for example, " $\widehat{G / H}$ is $H^{\perp}$."

$\mu=\mu_{G}$ and $v=v_{\widehat{G}}$ will denote Haar measures on $G$ and $\widehat{G}$, respectively. $L^{2}(G)$ is the space of square integrable functions on $G$, and the Fourier transform of $f \in L^{2}(G)$ is the function $\hat{f} \in L^{2}(\widehat{G})$ formally defined as

$$
\hat{f}(\gamma)=\int_{G} f(x) \overline{(x, \gamma)} d \mu(x),
$$

where $\gamma \in \widehat{G}$.

If the subgroup $H \subseteq G$ is open (and hence closed) and compact, then $H$ and $H^{\perp}$ are compact abelian groups, and the quotients $G / H$ and $\widehat{G} / H^{\perp}$ are discrete abelian groups. In that case, moreover, we can and shall make the following choices of normalization of Haar measure on each of the six groups:

- $\mu$ satisfies $\mu(H)=1$,

- $v$ satisfies $v\left(H^{\perp}\right)=1$,

- $\mu_{H}=\left.\mu\right|_{H}$,

- $v_{H^{\perp}}=\left.v\right|_{H^{\perp}}$,

- $\mu_{G / H}$ is counting measure, and

- $v_{\widehat{G} / H^{\perp}}$ is counting measure.

These choices guarantee that the Fourier transform is an isometry between $L^{2}(G)$ and $L^{2}(\widehat{G})$, and similarly between $L^{2}(H)$ and $L^{2}(\widehat{H})=L^{2}\left(\widehat{G} / H^{\perp}\right)$, and between $L^{2}(G / H)$ and $L^{2}(\widehat{G / H})=$ $L^{2}\left(H^{\perp}\right)$, e.g., [32, Section 31.1], [51]. The above normalizations are also compatible in the sense of Weil's formula (1.3), see Remark 1.1.

Extra attention about normalization is necessary in the verification of the aforementioned isometries in the case that $G$ is compact or discrete, see [32, Section 31.1] again; but this case does not arise herein, as noted in the discussion following Definition 2.5. On the other hand, we do have to deal with the case $G=\widehat{G}$; and some care is required, because the group $\widehat{G / H}$ may not coincide with the image of $H$ under the isomorphism $G \rightarrow \widehat{G}$. For example, if $G$ is the self-dual additive group of a finite extension $K$ of $\mathbb{Q}_{p}$, then $H^{\perp}=\widehat{G / H}$ may not be $H=\mathcal{O}_{K}$, but rather the inverse different of $K$; see, for example, [50, Section 7.1].

We shall sometimes suppress $\mu$ and $v$ in lengthy calculations; for example, we shall write " $d \gamma$ " instead of " $d v(\gamma)$ " in integrals. 
Let Aut $G$ denote the group under composition of homeomorphic automorphisms of $G$ onto itself. For any $A \in$ Aut $G$ and for any Borel set $U \subseteq G, \mu_{A}(U)=\mu(A U)$ defines a Haar measure on $G$. Formally,

$$
\int_{G} f \circ A^{-1}(x) d \mu(x)=\int_{G} f(x) d \mu_{A}(x) .
$$

Furthermore, $\mu_{A}$ cannot be the zero measure because $\mu(U)=\mu_{A}\left(A^{-1} U\right)$. Thus, there is a unique positive number $|A|$, the so-called modulus of $A$, with the property that for any measurable set $U \subseteq G$, we have $\mu_{A}(U)=|A| \mu(U)$. We shall use the following well-known fact, see [31, Section 15.26]:

$$
\forall A \in \text { Aut } G \text { and } \forall f \in C_{c}(G), \quad \int_{G} f \circ A(x) d x=|A|^{-1} \int_{G} f(x) d x .
$$

Also, every element $A \in$ Aut $G$ has an adjoint element $A^{*} \in$ Aut $\widehat{G}$, defined by $(A x, \gamma)=$ $\left(x, A^{*} \gamma\right)$ for all $x \in G$ and $\gamma \in \widehat{G}$. We have

- $\left(A^{*}\right)^{-1}=\left(A^{-1}\right)^{*}$,

- $|A|^{-1}=\left|A^{-1}\right|$, and

- $\left|A^{*}\right|=|A|$,

where the first equality is clear, the second is immediate from (1.1), and the third is by (1.1) again and the Plancherel theorem. Finally, and easily, the modulus function is a homomorphism Aut $G \longrightarrow(0, \infty)$, where $(0, \infty)$ is considered as a multiplicative group.

Let $\mathcal{A} \subseteq$ Aut $G$ be a nonempty countable subset of Aut $G$. In Theorem 4.2, $\mathcal{A}$ will be isomorphic to $\mathbb{Z}$; however, we can phrase the definitions and some results without assuming such a group structure.

For any $x \in G$, we write $[x]$ or $x+H$ for its image in $G / H$; that is, $[x]$ is the coset

$$
[x]=\{y \in G: y-x \in H\} \in G / H .
$$

Similarly, for $\gamma$ in $\widehat{G}$, we write $[\gamma]$ or $\gamma+H^{\perp}$ for its image in the quotient group $\widehat{G} / H^{\perp}$. If $([x], \gamma)_{G / H}$ denotes the action of the duality between $G / H$ and $H^{\perp}$, then $(x, \gamma)_{G}=([x], \gamma)_{G / H}$ for $x \in G, \gamma \in H^{\perp}$.

These and other standard facts about LCAGs that we shall use may be found in [31,32, 49, 51], and [53]. The standard facts from number theory that we shall use are found in [50] and [52], as well as [26, 35], and [55]. [23] and [46] are treatises on wavelet theory.

Remark 1.1. Classical Euclidean uniform sampling formulas depend essentially on periodization in terms of a discrete subgroup. Periodization induces a transformation from the given group to a compact quotient group, thereby allowing the analysis to be conducted in terms of Fourier series which lead to sampling formulas. The Shannon wavelet, which is a topic of Section 5.1, is associated with the simplest (and slowly converging) Classical Sampling Formula derived from the sinc function. Weil's formula,

$$
\int_{G} f(x) d \mu(x)=\int_{G / H}\left(\int_{H} f(x+y) d \mu_{H}(y)\right) d \mu_{G / H}(x),
$$

is a far reaching generalization of the idea of periodization, which itself is manifested in the term $\int_{H} f(x+y) d \mu_{H}(y)$. If two of the three Haar measures in (1.3) are given then the third can be 
normalized so that (1.3) is true on the space $C_{c}(G)$ of continuous functions with compact support. In our setting, with $\mu_{H}$ as the restriction of $\mu$ to $H$, the choice of $\mu_{G / H}$ to be counting measure is the appropriate normalization for (1.3). The analogous statement also applies to $v, v_{H^{\perp}}$, and $v_{\widehat{G} / H^{\perp}}$.

\section{Wavelets for groups with compact open subgroups}

\subsection{Translations}

Let $G$ be a LCAG with compact open subgroup $H \subseteq G$. $G$ may or may not contain a suitable discrete subgroup to serve as a lattice for translations, e.g., Example 2.10. One possible replacement for such a lattice is to choose a discrete $\operatorname{set} \mathcal{C}$ of coset representatives for the quotient $G / H$, and then translate elements of $L^{2}(G)$ by elements of $\mathcal{C}$. Kozyrev [36] used this strategy to show, for a certain choice $\mathcal{C}$ of coset representatives for $\mathbb{Q}_{p} / \mathbb{Z}_{p}$, that certain analogues of Haar wavelets are wavelets in $L^{2}\left(\mathbb{Q}_{p}\right)$ with respect to translations by elements of $\mathcal{C}$.

However, Kozyrev's examples are for the group $\mathbb{Q}_{p}$ only, see the discussion after Proposition 5.1. Moreover, there are substantial obstacles to generalizing Kozyrev's method to produce other wavelets, even for $\mathbb{Q}_{p}$. The main problem is that because $\mathcal{C}$ is not a group, there is no object to serve as the dual lattice in $\widehat{\mathbb{Q}}_{p}$. Thus, we shall need new translation operators on $L^{2}(G)$ which actually form a group.

Rather than choose elements $\mathcal{C}$ in $G$, we wish to construct one operator $\tau_{[s]}$ for each element $[s]$ of the discrete quotient group $G / H$, in such a way that $\tau_{[s]+[t]}=\tau_{[s]} \tau_{[t]}$, and so that $\tau_{[s]}$ is somehow similar to translation by $s$. Our operators will be determined by a choice $\mathcal{D}$ of coset representatives in $\widehat{G}$ for $\widehat{G} / H^{\perp}$. (See Remark 2.2 for more on $\mathcal{D}$.)

Definition 2.1. Let $G$ be a LCAG with compact open subgroup $H \subseteq G$. Let $\mathcal{D} \subseteq \widehat{G}$ be a set of coset representatives in $\widehat{G}$ for the quotient $\widehat{H}=\widehat{G} / H^{\perp}$.

(a) Define maps $\theta=\theta_{\mathcal{D}}: \widehat{G} \rightarrow \mathcal{D}$ and $\eta=\eta_{\mathcal{D}}: \widehat{G} \rightarrow H^{\perp} \subseteq \widehat{G}$ by

$$
\begin{aligned}
& \theta(\gamma)=\text { the unique } \sigma_{\gamma} \in \mathcal{D} \text { such that } \gamma-\sigma_{\gamma} \in H^{\perp} \subseteq \widehat{G}, \\
& \eta(\gamma)=\gamma-\theta(\gamma) .
\end{aligned}
$$

(b) For any fixed $[s] \in G / H$, define the following unimodular (hence $L^{\infty}$ ) weight function:

$$
w_{[s]}(\gamma)=w_{[s], \mathcal{D}}(\gamma)=\overline{\left(s, \eta_{\mathcal{D}}(\gamma)\right)} .
$$

(See Remark 2.3 for more on $w_{[s], \mathcal{D}}$.)

(c) For any fixed $[s] \in G / H$, define the multiplier $m_{[s]}$ on $L^{2}(\widehat{G})$ as multiplication by $w_{[s], \mathcal{D}}$, that is, for any $F \in L^{2}(\widehat{G})$,

$$
m_{[s]} F(\gamma)=m_{[s], \mathcal{D}} F(\gamma)=F(\gamma) w_{[s], \mathcal{D}}(\gamma) .
$$

(d) For any fixed $[s] \in G / H$ and for any $f \in L^{2}(G)$, define $\tau_{[s]} f$ to be the inverse transform of $m_{[s]} \widehat{f}$, that is,

$$
\tau_{[s]} f=\tau_{[s], \mathcal{D}} f=f * \check{w}_{[s], \mathcal{D}},
$$

where the pseudo-measure $\check{w}_{[s], \mathcal{D}}$ is the inverse Fourier transform of $w_{[s], \mathcal{D}}$.

Remark 2.2. (a) Recall that a set of coset representatives in $\widehat{G}$ for the quotient $\widehat{H}=\widehat{G} / H^{\perp}$ means a set $\mathcal{D} \subseteq \widehat{G}$ consisting of one element $\sigma \in \widehat{G}$ from each coset $\Sigma \in \widehat{G} / H^{\perp}$, i.e., 
$\Sigma=\sigma+H^{\perp}$. For example, if $\widehat{G}=\mathbb{R}$ and $H^{\perp}=\mathbb{Z}$, then the points of the interval $[0,1) \subseteq \mathbb{R}$ are a choice of coset representatives for the quotient $\mathbb{R} / \mathbb{Z}$.

(b) In our setting, the quotient $\widehat{H}=\widehat{G} / H^{\perp}$ is discrete, and therefore $\mathcal{D}$ is also a discrete subset of $\widehat{G}$. However, just as $[0,1)$ is not a subgroup of $\mathbb{R}$, our $\mathcal{D}$ is not necessarily a subgroup of $\widehat{G}$. In fact, groups such as $\widehat{G}=\mathbb{Q}_{p}$ (with compact open subgroup $H^{\perp}=\mathbb{Z}_{p}$ ) have no nontrivial discrete subgroups, and therefore cannot have a $\mathcal{D}$ which forms a group.

On the other hand, the image $[\mathcal{D}]=\{[\sigma]: \sigma \in \mathcal{D}\}$ of $\mathcal{D}$ in the quotient $\widehat{G} / H^{\perp}$ is the full group $\widehat{G} / H^{\perp}=\widehat{H}$, because $\mathcal{D}$ contains one element from every coset.

(c) Just as the choice of $[0,1)$ as a subset of $\mathbb{R}$ is no more natural than the choice of $(-1 / 2,1 / 2]$, there is usually not a natural or canonical choice of the set $\mathcal{D}$. Nevertheless, in most cases, we can construct a set $\mathcal{D}$ without using the axiom of choice, e.g., Section 2.3 and Lemma 2.7 below.

Remark 2.3. (a) As the notation suggests, $w_{[s], \mathcal{D}}$ depends on $\mathcal{D}$ and on the coset $[s] \in G / H$, but it does not depend on the particular element $s \in G$. Indeed, if $s, t \in G$, then

$$
w_{[s+t], \mathcal{D}}(\gamma)=\overline{(s+t, \eta(\gamma))}=\overline{(s, \eta(\gamma))(t, \eta(\gamma))}=w_{[s], \mathcal{D}}(\gamma) w_{[t], \mathcal{D}}(\gamma) .
$$

The claim that $w_{[s], \mathcal{D}}$ is independent of the particular choice of $s$ in a given coset now follows from the observation that if $t \in H$, then $w_{[t], \mathcal{D}}=1$. It is also immediate that our operators have the desired property that $\tau_{[s]+[t]}=\tau_{[s]} \tau_{[t]}$.

(b) The inverse Fourier transform of $w_{[s], \mathcal{D}}$ is a pseudo-measure $\check{w}_{[s], \mathcal{D}}$ on $G$; see [7] for pseudo-measures which are defined as tempered distributions whose Fourier transforms are elements of $L^{\infty}(\widehat{G})$. In particular, the convolution $f * \check{w}_{[s], \mathcal{D}}$ in the definition of $\tau_{[s], \mathcal{D}}$ is well defined by the action of the Fourier transform, viz.,

$$
{\widehat{f * \check{w}_{[s], \mathcal{D}}}}=\widehat{f} w_{[s], \mathcal{D}},
$$

which, in turn, is well-defined since $\widehat{f} w_{[s], \mathcal{D}} \in L^{2}(\widehat{G})$. Note that the operators $m_{[s], \mathcal{D}}$ and $\tau_{[s], \mathcal{D}}$ are unitary because $w_{[s], \mathcal{D}}$ is unimodular.

Remark 2.4. In our analogy with wavelets on $\mathbb{R}^{d}$, $[s]$ takes the place of a lattice element $n$. Similarly, the pseudo-measure $\breve{w}_{[s], \mathcal{D}}$ takes the place of the $\delta$ measure at $n$. Indeed, the $\delta$ measure at $s \in G$ acts on $L^{2}(\widehat{G})$ by multiplication by $\overline{(s, \gamma)}$, while $m_{[s]}$ acts by multiplication by $\overline{(s, \eta(\gamma))}$. The ratio of the two is $(s, \theta(\gamma))$, which has absolute value 1 everywhere.

In the Euclidean setting, $G / H$ and $\mathcal{D}$ are both analogous to the discrete subgroup $\mathbb{Z}^{d}$, so that both $s$ and $\theta(\gamma)$ would be elements of $\mathbb{Z}^{d}$. In particular, the $(s, \theta)$ factor would be identically 1. Thus, we may consider our operators $\tau_{[s]}$ to be as legitimate an analogue of the classical Euclidean translation operators as are the naive translation-by-s operators.

In fact, if $s \in H$, then $w_{[s], \mathcal{D}}=1$, so that $\check{w}_{[s], \mathcal{D}}$ is the $\delta$ measure at 0 , and therefore $f * \check{w}_{[s], \mathcal{D}}=f$. Generally, for $s \notin H, \check{w}_{[s], \mathcal{D}}$ is more complicated, but the action of $\tau_{[s]}$ is still easily computable, see [14].

\subsection{Expansive automorphisms and dilations}

When constructing wavelets in $L^{2}\left(\mathbb{R}^{d}\right)$, one would like an automorphism (such as multiplication-by-2) which is expansive. This means that the automorphism must not only map the lattice into itself, but it must have all eigenvalues greater than one in absolute value. Although our group 
$G$ may have neither a lattice nor a suitable notion of eigenvalues of automorphisms, we can state a reasonable analogue of expansiveness as follows.

Definition 2.5. Let $G$ be a LCAG with compact open subgroup $H \subseteq G$, and let $A \in$ Aut $G$. We say that $A$ is expansive with respect to $H$ if both of the following conditions hold:

(a) $H \subsetneq A H$, and

(b) $\bigcap_{n \leq 0} A^{n} H=\{0\}$.

The inclusion condition of expansiveness is the analogue of mapping the lattice into itself as in the definition of an MRA. Similarly, the intersection condition is the analogue of having eigenvalues greater than 1 , and a similar condition in $\mathbb{R}^{d}$ is a consequence of the other properties in the definition of an MRA, e.g., [44].

Note that if $A$ is an expansive automorphism of $G$, then $|A|$ is an integer greater than 1 , just as is true of an expansive integer matrix. Indeed, $H$ is a proper subgroup of $A H$, so that $A H$ may be covered by (disjoint) cosets $s+H$. Each coset has measure 1, so that $A H$ must have measure equal to the number of cosets. Thus, $|A|=\mu(A H)$ is just the number of elements in the finite quotient group $(A H) / H$.

Further note that if $A$ is expansive, then $\mu(G)=\infty$. To see this, simply observe that by applying the first condition of Definition 2.5 repeatedly, we have

$$
\mu(G) \geq \mu\left(A^{n} H\right)=|A|^{n} \mu(H)=|A|^{n} \geq 2^{n}
$$

for every integer $n$. As a result, $G$ cannot be compact, and $G / H$ is infinite.

We may alternatively characterize expansiveness by the action of the adjoint automorphism $A^{*}$ on $\widehat{G}$, as the following lemma shows. Note that as a consequence of this lemma, if $G$ has an expansive automorphism $A$, then $\widehat{G}$ is $\sigma$-compact. In addition, $\widehat{G}$ cannot be compact, so $G$ cannot be discrete.

Lemma 2.6. Let $G$ be a $L C A G$ with compact open subgroup $H \subseteq G$, and let $A \in$ Aut $G$.

(a) $H \subseteq A H$ if and only if $H^{\perp} \subseteq A^{*} H^{\perp}$.

(b) $H \subsetneq A H$ if and only if $H^{\perp} \subsetneq A^{*} H^{\perp}$.

(c) Suppose $H \subseteq A H$. Then

$$
\bigcap_{n \leq 0} A^{n} H=\{0\} \Longleftrightarrow \bigcup_{n \geq 0} A^{* n} H^{\perp}=\widehat{G} .
$$

Proof. (a) Suppose $H \subseteq A H$, and consider $\gamma \in H^{\perp}$. For any $x \in H$, we have $x \in A H$, and therefore $x=A y$ for some $y \in H$. Hence,

$$
\left(x,\left(A^{*}\right)^{-1} \gamma\right)=\left(A y,\left(A^{*}\right)^{-1} \gamma\right)=(y, \gamma)=1 .
$$

Thus, $\left(A^{*}\right)^{-1} \gamma \in H^{\perp}$, and so $\gamma \in A^{*} H^{\perp}$. It follows that $H^{\perp} \subseteq A^{*} H^{\perp}$. The converse is similar.

(b) If $H \subsetneq A H$, then $|A|>1$, and so $A^{*} H^{\perp} \neq H^{\perp}$, because $v\left(A^{*} H^{\perp}\right)=\left|A^{*}\right|=|A|>$ $1=v\left(H^{\perp}\right)$. Again, the converse is similar.

(c) ( $\Longleftarrow$ ) Pick any $x \in \bigcap_{n \leq 0} A^{n} H$, that is, any $x \in G$ with $A^{n} x \in H$ for all $n \geq 0$. For any $\gamma \in \widehat{G}$, there is an integer $n \geq 0$ such that $\gamma \in\left(A^{*}\right)^{n} H^{\perp}$, by assumption. Thus,

$$
(x, \gamma)=\left(A^{n} x,\left(A^{*}\right)^{-n} \gamma\right)=1 \text {, }
$$


because $A^{n} x \in H$ and $\left(A^{*}\right)^{-n} \gamma \in H^{\perp}$. Since $(x, \gamma)=1$ for every $\gamma \in \widehat{G}$, we must have $x=0$.

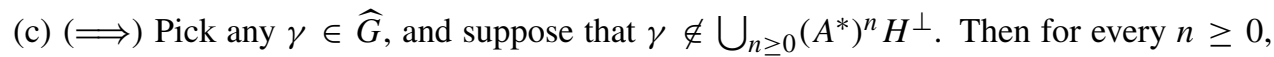
there is some $x_{n} \in A^{-n} H$ such that $\zeta_{n}=\left(x_{n}, \gamma\right) \neq 1$. Thus, there is an integer $m \geq 1$ such that $\zeta_{n}^{m}$ has negative real part. Let $y_{n}=m x_{n}$, so that $y_{n} \in A^{-n} H$ (because $A^{-n} H$ is a group) and $\operatorname{Re}\left(y_{n}, \gamma\right)<0$.

We claim that the sequence $\left\{y_{n}\right\}$ has an accumulation point at $0 \in \widehat{G}$. Certainly some accumulation point exists, because $y_{n} \in A^{-n} H \subseteq H$ and $H$ is compact. If 0 is not an accumulation point, then there is a nonzero accumulation point $y$. Since $y \neq 0$, we have $y \notin \bigcap A^{-n} H$, so there must be an integer $N \geq 0$ for which $y \notin A^{-N} H$. For any $n \geq N$, we have $A^{-n} H \subseteq A^{-N} H$; therefore $y \notin A^{-n} H$. By the previous paragraph, then, $y+A^{-N} H$ is an open neighborhood of $y$ containing only finitely many $y_{n}$. Hence, $y \neq 0$ is not actually an accumulation point, and so the only accumulation point of $\left\{y_{n}\right\}$ is $y=0$.

Let $U=\{x \in H: \operatorname{Re}(x, \gamma)>0\}$. The map $(\cdot, \gamma): G \rightarrow \mathbb{C}$ is continuous by definition, and therefore $U$ is open. Clearly $0 \in U$. Thus, there must be infinitely many $y_{n}$ in $U$. However, $\operatorname{Re}\left(y_{n}, \gamma\right)<0$, and so $y_{n} \notin U$. That is a contradiction, and therefore $\gamma$ must lie in $\bigcup_{n \geq 0}\left(A^{*}\right)^{n} H^{\perp}$.

In Remark 2.2 (c), it was noted that a set $\mathcal{D}$ of coset representatives for an infinite quotient $\widehat{G} / H^{\perp}$ can usually be constructed without using the axiom of choice. In fact, given an expansive automorphism $A$ and a choice of coset representatives for the finite quotient $H^{\perp} /\left(\left(A^{*}\right)^{-1} H^{\perp}\right)$, we can generate such a $\mathcal{D}$ by the following lemma.

Lemma 2.7. Let $G$ be a $L C A G$ with compact open subgroup $H \subseteq G$. Let $A \in$ Aut $G$ be an expansive automorphism with modulus $|A|=N \geq 2$, and let $\mathcal{D}_{1}=\left\{\rho_{0}, \ldots, \rho_{N-1}\right\}$ be a set of coset representatives for the quotient $H^{\perp} /\left(\left(A^{*}\right)^{-1} H^{\perp}\right)$, with $\rho_{0}=0$. Define $\mathcal{D} \subseteq \widehat{G}$ to be the set of all elements $\sigma \in \widehat{G}$ of the form

$$
\sigma=\sum_{j=1}^{n}\left(A^{*}\right)^{j} \rho_{i_{j}}, \quad \text { where } n \geq 1 \text { and } i_{j} \in\{0,1, \ldots, N-1\} \text {. }
$$

Then $\mathcal{D}$ is a set of coset representatives for the quotient $\widehat{G} / H^{\perp}$. Moreover, $A^{*} \mathcal{D} \subsetneq \mathcal{D}$.

Proof. First, we need to show that if $\sigma, \sigma^{\prime} \in \mathcal{D}$ with $\sigma-\sigma^{\prime} \in H^{\perp}$, then $\sigma=\sigma^{\prime}$. Write

$$
\sigma=\sum_{j=1}^{n}\left(A^{*}\right)^{j} \rho_{i_{j}}, \quad \sigma^{\prime}=\sum_{j=1}^{n^{\prime}}\left(A^{*}\right)^{j} \rho_{i_{j}^{\prime}},
$$

and assume without loss that $n \geq n^{\prime}$. Thus, we have

$$
\sum_{j=1}^{n^{\prime}}\left(A^{*}\right)^{j}\left(\rho_{i_{j}}-\rho_{i_{j}^{\prime}}\right)+\sum_{j=n^{\prime}+1}^{n}\left(A^{*}\right)^{j} \rho_{i_{j}} \in H^{\perp} .
$$

If $\sigma \neq \sigma^{\prime}$, then let $k$ be the largest value of $j$ for which the $\left(A^{*}\right)^{j}$ term is nonzero. Note that every other term is of the form either $\left(A^{*}\right)^{j}\left(\rho_{i_{j}}-\rho_{i_{j}^{\prime}}\right) \in\left(A^{*}\right)^{j} H^{\perp}$ or $\left(A^{*}\right)^{j} \rho_{i_{j}} \in\left(A^{*}\right)^{j} H^{\perp}$, with $j \leq k-1$. Since $\left(A^{*}\right)^{j} H^{\perp} \subseteq\left(A^{*}\right)^{k-1} H^{\perp}$, we can multiply both sides of the inclusion (2.5) by $\left(A^{*}\right)^{-k}$ to obtain

$$
\rho_{i_{k}}-\rho_{i_{k}^{\prime}} \in\left(A^{*}\right)^{-1} H^{\perp}
$$


if $k \leq n^{\prime}$, or

$$
\rho_{i_{k}} \in\left(A^{*}\right)^{-1} H^{\perp}
$$

if $k>n^{\prime}$. However, either of those conclusions implies that the $\left(A^{*}\right)^{k}$ term of (2.5) was zero, which contradicts our assumption that $\sigma \neq \sigma^{\prime}$. Thus, $\sigma=\sigma^{\prime}$, as desired.

Second, given $\gamma \in \widehat{G}$, we need to show that $\gamma \in \sigma+H^{\perp}$ for some $\sigma \in \mathcal{D}$. Let $n$ be the smallest nonnegative integer such that $\left(A^{*}\right)^{-n} \gamma \in H^{\perp}$. Such an integer must exist, by Lemma 2.6 (c). We claim that $\gamma$ is in $\sigma+H^{\perp}$ for some $\sigma$ of the form

$$
\sigma=\sum_{j=1}^{n}\left(A^{*}\right)^{j} \rho_{i_{j}} .
$$

(Note that the $n$ in the sum is the same as the $n$ we have just selected.) We proceed by induction on $n$. If $n=0$, simply let $\sigma=0 \in \mathcal{D}$, and we get $\gamma \in H^{\perp}=\sigma+H^{\perp}$. For general $n \geq 1$, let $\rho_{i_{n}}$ be the element of $\mathcal{D}_{1}$ which is in the same coset of $\left(A^{*}\right)^{-1} H^{\perp}$ as $\left(A^{*}\right)^{-n} \gamma$. Then $\left(A^{*}\right)^{-(n-1)}\left(\gamma-\left(A^{*}\right)^{n} \rho_{i_{n}}\right) \in H^{\perp}$, and our induction is complete.

Finally, the fact that $A^{*} \mathcal{D} \subseteq \mathcal{D}$ is clear from the form of $\sigma \in \mathcal{D}$ and the fact that $0 \in \mathcal{D}_{1}$; and $A^{*} \mathcal{D} \neq \mathcal{D}$ because $A^{*} \rho_{1} \in \mathcal{D} \backslash A^{*} \mathcal{D}$. follows.

We define dilation operators $\delta_{A}$ (not to be confused with the $\delta$ measure) in the usual way, as

Definition 2.8. Let $G$ be a LCAG, and let $A \in$ Aut $G$. Define an operator $\delta_{A}$ on $L^{2}(G)$ by

$$
\forall f \in L^{2}(G), \quad \delta_{A} f(x)=|A|^{1 / 2} f(A x) .
$$

Definition 2.9. Let $G$ be a LCAG with compact open subgroup $H \subseteq G$, let $\mathcal{D}$ be a choice of coset representatives in $\widehat{G}$ for $\widehat{H}=\widehat{G} / H^{\perp}$, let $A \in$ Aut $G$, and consider $[s] \in G / H$. The dilated translate of $f \in L^{2}(G)$ is defined as

$$
f_{A,[s]}(x)=\delta_{A} \tau_{[s], \mathcal{D}} f(x)=|A|^{1 / 2} \cdot\left(f * \check{w}_{[s], \mathcal{D}}\right)(A x) .
$$

We compute

$$
\widehat{f_{A,[s]}}(\gamma)=|A|^{-1 / 2} \widehat{f}\left(\left(A^{*}\right)^{-1} \gamma\right) \overline{\left(s, \eta\left(\left(A^{*}\right)^{-1} \gamma\right)\right)} .
$$

\subsection{Examples}

The conditions that a LCAG $G$ contain a compact open subgroup $H$ and have an expansive automorphism $A$ place strong constraints on the topologies of $G$ and $\widehat{G}$. Such groups $G$ are a subclass of all totally disconnected groups.

Figure 1 indicates the structure of $\widehat{G}$ for these particular totally disconnected groups $G$ in the case $|A|=3$. First note that $H^{\perp} \subsetneq A^{*} H^{\perp}$ by Lemma 2.6 (b). If $\sigma_{1} \in A^{*} H^{\perp} \backslash H^{\perp}$ then $H^{\perp} \bigcap\left(\sigma_{1}+H^{\perp}\right)=\emptyset$. Continuing this procedure, we use a compactness argument to assert the existence of finitely many cosets $\sigma+H^{\perp}$ covering $A^{*} H^{\perp}$, and this finite number is $|A|$ which is the order of $A^{*} H^{\perp} / H^{\perp}$. Thus, we see that $A^{*} H^{\perp}$ is the finite union $H \bigcup\left(\sigma_{1}+H^{\perp}\right) \bigcup\left(\sigma_{2}+H^{\perp}\right)$ in Figure 1. The remaining circles in Figure 1 follow by considering $A^{*} H^{\perp} \subsetneq\left(A^{*}\right)^{2} H^{\perp}$ and $\left(A^{*}\right)^{-1} H^{\perp} \subsetneq A^{*} H^{\perp}$. The idea of the diagram is that the self-similar pattern of nested circles continues forever. 


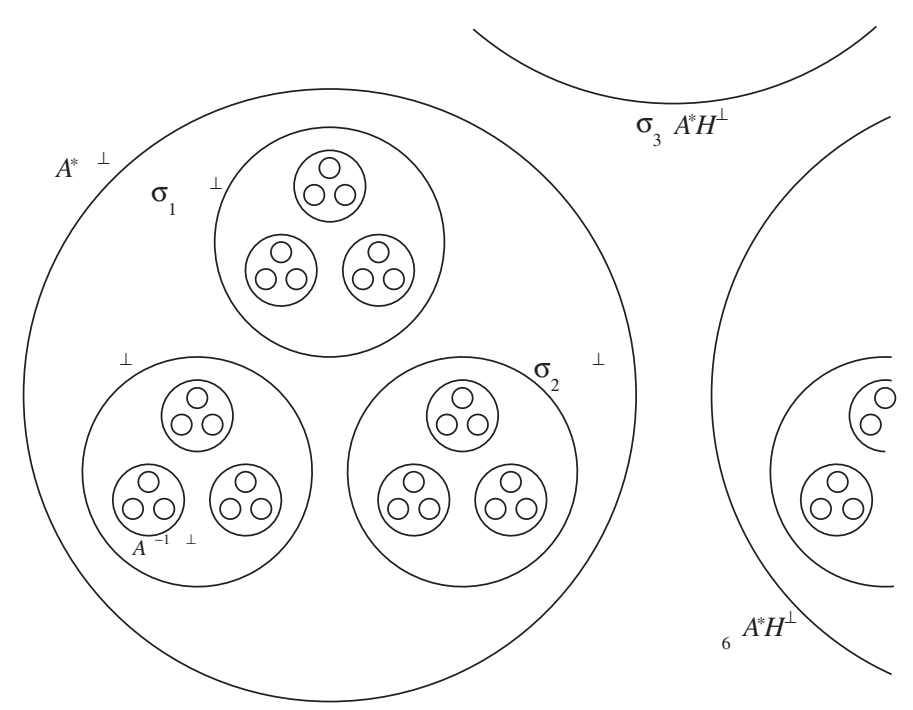

FIGURE $1 \widehat{G}$ for a LCAG with compact open subgroup $H$ and expansive automorphism $A$, with $|A|=3$.

$\widehat{G}$ is an infinite disjoint union of translates of $H^{\perp}$. In Figure $1, H^{\perp}$ looks very much like a Cantor set with constant rate of dissection determined by $|A|$; thus, it is well to point out that $\widehat{G}$ need not be totally disconnected.

In spite of the strong constraints mentioned above, many such groups $G$ and expansive automorphisms $A$ exist; and many have applications in number theory. We now give some examples and comment on their translation and dilation operators.

Example 2.10. Let $p \geq 2$ be a prime number. The $p$-adic field $\mathbb{Q}_{p}$ consists of all formal Laurent series in $p$ with coefficients $0,1, \ldots, p-1$. Thus,

$$
\mathbb{Q}_{p}=\left\{\sum_{n \geq n_{0}} a_{n} p^{n}: n_{0} \in \mathbb{Z} \text { and } a_{n} \in\{0,1, \ldots, p-1\}\right\},
$$

with addition and multiplication as usual for Laurent series, except with carrying of digits, so that, for example, in $\mathbb{Q}_{5}$, we have

$$
(3+2 \cdot 5)+(4+3 \cdot 5)=2+1 \cdot 5+1 \cdot 5^{2} .
$$

(Equivalently, $\mathbb{Q}_{p}$ is the completion of $\mathbb{Q}$ with respect to the $p$-adic absolute value $\left|p^{r} x\right|_{p}=p^{-r}$ for all $r \in \mathbb{Z}$ and all $x \in \mathbb{Q}$ such that the numerator and denominator of $x$ are both relatively prime to $p$.) Then $G=\mathbb{Q}_{p}$ is a LCAG under addition, with topology induced by $|\cdot|_{p}$, and with compact open subgroup $H=\mathbb{Z}_{p}$ consisting of Taylor series in $p$; equivalently, $\mathbb{Z}_{p}$ is the closure of $\mathbb{Z} \subseteq \mathbb{Q}_{p}$.

$\mathbb{Q}_{p}$ is self-dual, with duality action given by $(x, \gamma)=\chi(x \gamma)$, where $\chi: \mathbb{Q}_{p} \rightarrow \mathbb{C}$ is the character given by

$$
\chi\left(\sum_{n \geq n_{0}} a_{n} p^{n}\right)=\exp \left(2 \pi i \sum_{n=n_{0}}^{-1} a_{n} p^{n}\right) .
$$

The annihilator $\mathbb{Z}_{p}^{\perp}$ is just $\mathbb{Z}_{p}$ under this self-duality.

The quotient $\mathbb{Q}_{p} / \mathbb{Z}_{p}$ is isomorphic to $\mu_{p^{\infty}}$, the subgroup of $\mathbb{C}^{\times}$consisting of all roots of unity $\zeta$ for which $\zeta^{p^{n}}=1$ for some $n \geq 0$. One possible choice for $\mathcal{D}$, and the one used by 
Kozyrev [36] in $G$ rather than in $\widehat{G}$, is

$$
\mathcal{D}_{\mathrm{Koz}}=\left\{\sum_{n=n_{0}}^{-1} a_{n} p^{n}: n_{0} \leq-1 \text { and } a_{n}=0,1, \ldots, p-1\right\},
$$

which is not a subgroup. (Equivalently, $\mathcal{D}_{\mathrm{Koz}}$ is the set of all rational numbers of the form $m / p^{n}$ for which $0 \leq m \leq p^{n}-1$.)

A natural choice for $A$ would be multiplication-by- $1 / p$, which takes $\mathbb{Z}_{p}$ to the larger subgroup $p^{-1} \mathbb{Z}_{p}$; it is easy to verify that this map is expansive. More generally, for any nonzero $a \in \mathbb{Q}_{p}$, the multiplication-by- $a$ map is an automorphism of the additive group $\mathbb{Q}_{p}$, with adjoint map also given by multiplication-by- $a$ on the dual $\mathbb{Q}_{p}$. In fact, the only automorphisms of $\mathbb{Q}_{p}$ are the multiplication-by- $a$ maps; and these maps are expansive if and only if $|a|_{p}>1$.

Example 2.11. Let $p \geq 2$ be a prime number, and let $\mathbb{F}_{p}$ denote the field of order $p$, with elements $\{0,1, \ldots, p-1\}$. The field $\mathbb{F}_{p}((t))$ consists of formal Laurent series in the formal variable $t$, that is,

$$
\mathbb{F}_{p}((t))=\left\{\sum_{n \geq n_{0}} a_{n} t^{n}: n_{0} \in \mathbb{Z} \text { and } a_{n} \in \mathbb{F}_{p}\right\},
$$

with the usual addition and multiplication of Laurent series, but this time without carrying. For example, in $\mathbb{F}_{5}((t))$, we have

$$
(3+2 \cdot t)+(4+3 \cdot t)=2 .
$$

(Equivalently, $\mathbb{F}_{p}((t))$ is the completion of the field $\mathbb{F}_{p}(t)$ of rational functions with coefficients in $\mathbb{F}_{p}$ with respect to the absolute value $|f|_{0}=p^{-\operatorname{ord}_{0}(f)}$, where $\operatorname{ord}_{0}(f)$ is the order of the zero (or negative the order of the pole) of $f$ at 0 ). Then $G=\mathbb{F}_{p}((t))$ is a LCAG under addition, with the topology induced by $|\cdot|_{0}$, and with compact open subgroup $H=\mathbb{F}_{p}[[t]]$ consisting of Taylor series in $t$; equivalently, $H$ is the closure of the ring of polynomials $\mathbb{F}_{p}[t] \subseteq \mathbb{F}_{p}((t))$.

$G$ is self-dual, with duality action given by $(x, \gamma)=\chi(x \gamma)$, where $\chi: G \rightarrow \mathbb{C}$ is the character given by

$$
\chi\left(\sum_{n \geq n_{0}} a_{n} t^{n}\right)=\exp \left(2 \pi i a_{-1} p^{-1}\right) .
$$

The annihilator $H^{\perp}$ is just $H$ under this self-duality.

The quotient $G / H$ is isomorphic to a countable direct sum of copies of $\mathbb{F}_{p}$ (one copy for each negative power of $t$ ). In fact,

$$
\Lambda_{\mathbb{F}_{p}((t))}=\left\{\sum_{n=n_{0}}^{-1} a_{n} t^{n}: n_{0} \leq-1 \text { and } a_{n} \in \mathbb{F}_{p}\right\}
$$

is a discrete subgroup of $G$, and $G=H \times \Lambda_{\mathbb{F}_{p}((t))}$ in the obvious way. (The group $\Lambda_{\mathbb{F}_{2}((t))}$ is the lattice used by Lang $[39,40,41]$ to study wavelets on $G=\mathbb{F}_{2}((t))$, which is sometimes called the Cantor dyadic group.) $\Lambda_{\mathbb{F}_{p}((t))}$, viewed as a subset of $\widehat{G}$, is a logical choice for our $\mathcal{D}$; in that case, because $\mathcal{D}$ is actually a group, the operators $\tau_{[s], \mathcal{D}}$ are in fact the usual translation operators

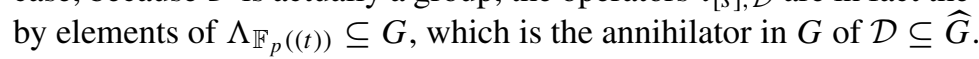

One natural choice for $A$ would be multiplication-by-1/t, which takes $H$ to the larger subgroup $t^{-1} H$. As before, for any nonzero $a \in \mathbb{Q}_{p}$, the multiplication-by- $a$ map is an automorphism 
of the additive group $G$, with adjoint also multiplication-by- $a$; and it is expansive if and only if $|a|_{0}>1$. However, in contrast to $\mathbb{Q}_{p}$, many other automorphisms of $\mathbb{F}_{p}((t))$ exist, because the coefficients of the different powers of $t$ are arithmetically independent of one another. For example, one could define an automorphism which acts (non expansively) only by multiplying the $t^{-17}$ coefficient by some integer $m$ not divisible by $p$.

Example 2.12. Let $L$ be any field which is a finite extension of either $K=\mathbb{Q}_{p}$ or $K=\mathbb{F}_{p}((t))$. Then the absolute value on $K$ extends in a unique way to $L$. The ring of integers $\mathcal{O}_{L}$ consists of all elements of $L$ which are roots of monic polynomials with coefficients in $\mathcal{O}_{K}$, where $\mathcal{O}_{K}$ is $\mathbb{Z}_{p}$ if $K=\mathbb{Q}_{p}$ or $\mathbb{F}_{p}[[t]]$ if $K=\mathbb{F}_{p}((t))$. Then $G=L$ is a LCAG under addition, with compact open subgroup $H=\mathcal{O}_{L}$. We may choose an element $\pi_{L} \in \mathcal{O}_{L}$ of largest possible absolute value strictly less than 1 ; such an element, called a uniformizer of $L$, always exists, and it is analogous to $t \in \mathbb{F}_{p}((t))$ or $p \in \mathbb{Q}_{p}$. The quotient $\mathcal{O}_{L} /\left(\pi_{L} \mathcal{O}_{L}\right)$ is a field of order $p^{s}$, for some integer $s \geq 1$.

$G$ is self-dual, with duality action given by $(x, \gamma)=\chi(\operatorname{Tr}(x \gamma))$, where $\chi: K \rightarrow \mathbb{C}$ is as in the preceding examples, and $\operatorname{Tr}$ is the trace map from $L$ to $K$. This time, the annihilator $H^{\perp}$, known as the inverse different of $L$, may be larger than $\mathcal{O}_{L}$, and it is of the form $\pi_{L}^{-r} \mathcal{O}_{L}$ for some integer $r \geq 0$.

If $\mathcal{D}_{1}$ is a list of $p^{s}$ coset representatives for the finite quotient $\mathcal{O}_{L} /\left(\pi_{L} \mathcal{O}_{L}\right)$, then

$$
\mathcal{D}=\left\{\sum_{n=n_{0}}^{-r-1} a_{n} \pi_{L}^{n}: n_{0} \leq-r-1 \text { and } a_{n} \in \mathcal{D}_{1}\right\}
$$

is a set of coset representatives for $\widehat{G} / H^{\perp}$, by Lemma 2.7. If $K=\mathbb{Q}_{p}$, then $\mathcal{D}$ cannot be a group, as noted in Example 2.10; but if $K=\mathbb{F}_{p}((t))$, then we can choose $\mathcal{D}_{1}$ to be a group, in which case $\mathcal{D}$ is also a group $\Lambda$, as in Example 2.11.

As before, for any nonzero $a \in L$, the multiplication-by- $a$ map is an automorphism of $L$ (expansive if and only if $|a|>1$ ), with adjoint also given by multiplication-by- $a$. For extensions of $\mathbb{Q}_{p}$, a few other automorphisms involving Galois actions are possible; for extensions of $\mathbb{F}_{p}((t))$, many more automorphisms are again possible.

Example 2.13. Let $G_{1} \ldots, G_{N}$ be LCAGs with compact open subgroups $H_{1}, \ldots, H_{N}$ and coset representatives $\mathcal{D}_{i}$ for each quotient $\widehat{G}_{i} / H_{i}^{\perp}$. Then $G=G_{1} \times \cdots \times G_{N}$ is a LCAG with compact open subgroup $H=H_{1} \times \cdots \times H_{N}$ and coset representatives $\mathcal{D}=\mathcal{D}_{1} \times \cdots \times \mathcal{D}_{N}$. Thus, for example, $\mathbb{Q}_{p}^{N}$ is an LCAG with compact open subgroup, as is any product of fields from the preceding examples.

An automorphism $A$ of $\mathbb{Q}_{p}^{N}$ may be represented by a matrix in $G L\left(N, \mathbb{Q}_{p}\right)$, just as automorphisms of $\mathbb{R}^{N}$ are matrices in $G L(N, \mathbb{R})$. As is the case over $\mathbb{R}$, the adjoint automorphism is simply represented by the transpose of the original matrix. Such a matrix map is expansive if and only if its eigenvalues have absolute value $\left(|\cdot|_{p}\right)$ greater than 1.

Example 2.14. Let $X$ be a LCAG with compact open subroup $Y$, let $W$ be a nontrivial discrete abelian group, and set $G=X \times W$. Then $G$ is a LCAG with a compact open subgroup $H=$ $Y \times\{0\}$. Note that if $W$ is infinite, then, even if $X$ is self-dual, the dual group $\widehat{G}=\widehat{X} \times \widehat{W}$ need not be isomorphic to $G$.

Furthermore, the annihilator $H^{\perp}$ is $Y^{\perp} \times \widehat{W}$. Thus, if $A_{X}$ is an automorphism of $X$ which is expansive with respect to $Y$, then $A=A_{X} \times \mathrm{id}_{W}$ is an automorphism of $G$ which is expansive with respect to $H$. After all, the adjoint $A^{*}$ acts as $A_{X}^{*} \times \mathrm{id}_{\widehat{W}}$, so that the union of all positive 
iterates $\left(A^{*}\right)^{n} H^{\perp}$ is $\widehat{G}$. This occurs in spite of the fact that the iterates $A^{n} H$ together cover only $X \times\{0\}$, rather than all of $G$.

Example 2.15. If $\mathbf{a}=\left\{a_{n}\right\}_{n \in \mathbb{Z}} \subseteq \mathbb{N}$ is a bi-infinite sequence of positive integers, one may define a group of a-adics as formal Laurent series in $t$ of the form

$$
G=G_{\mathbf{a}}=\left\{\sum_{n \geq n_{0}} c_{n} t^{n}: n_{0} \in \mathbb{Z} \text { and } c_{n} \in\left\{0,1, \ldots, a_{n}-1\right\}\right\},
$$

with usual addition of Laurent series, but with carrying of digits, as in $\mathbb{Q}_{p}$; see [31, Section 10.2]. (Thus, $\mathbb{Q}_{p}$ is the special case of $G_{\mathbf{a}}$ with $a_{n}=p$ for all $n$.) Let $H=H_{\mathbf{a}}$ be the subgroup consisting of Taylor series. Then $G$ is a LCAG with compact open subgroup $H$. As before, we could let $\mathcal{D}$ consist of all Laurent series for which all nonnegative power terms have coefficient 0 .

In this case, aside from uniform situations such as $\mathbb{Q}_{p}$ for which each $a_{n}$ is the same, $G$ generally has no expansive isomorphisms, because the different powers of $t$ have incompatible coefficients. Of course, one could fix an integer $m$ and define $a_{n}=m$ for all $n$, so that multiplication-by- $1 / m$ would be an expansive automorphism of the resulting group $G_{\mathbf{a}}$. Then by the Chinese Remainder Theorem, $G_{\mathbf{a}}$ would be isomorphic to a product $\mathbb{Q}_{p_{1}} \times \cdots \times \mathbb{Q}_{p_{s}}$, as $p_{i}$ ranges over the distinct prime factors of $m$; such groups have therefore already been discussed in the preceding examples.

\section{Theory of wavelet sets}

\subsection{Wavelets and $(\tau, \mathcal{D})$-congruence}

Now that we have appropriate dilation and translation operators, we are prepared to define wavelets on our group $G$.

Definition 3.1. Let $G$ be a LCAG with compact open subgroup $H \subseteq G$, let $\mathcal{D} \subseteq \widehat{G}$ be a choice of coset representatives in $\widehat{G}$ for $\widehat{H}=\widehat{G} / H^{\perp}$, and let $\mathcal{A} \subseteq$ Aut $G$ be a countable nonempty set of automorphisms of $G$. Consider $\Psi=\left\{\psi_{1}, \ldots, \psi_{N}\right\} \subseteq L^{2}(G)$. $\Psi$ is a set of wavelet generators for $L^{2}(G)$ with respect to $\mathcal{D}$ and $\mathcal{A}$ if

$$
\left\{\psi_{j, A,[s]}: 1 \leq j \leq N, A \in \mathcal{A},[s] \in G / H\right\}
$$

forms an orthonormal basis for $L^{2}(G)$, where

$$
\psi_{j, A,[s]}(x)=\delta_{A} \tau_{[s]} \psi_{j}(x)=|A|^{1 / 2}\left(\tau_{[s], \mathcal{D}} \psi_{j}\right)(A x),
$$

as in Equation (2.7). In that case, the resulting basis is called a wavelet basis for $L^{2}(G)$.

If $\Psi=\{\psi\}$, then $\psi$ is a single wavelet for $L^{2}(G)$.

The wavelets $\psi$ that we construct in this article will have the property that $\widehat{\psi}$ is the characteristic function of some measurable subset of $\widehat{G}$. We therefore state the following definition, e.g., $[19,20]$.

Definition 3.2. Let $G, H, \mathcal{D}$, and $\mathcal{A}$ be as in Definition 3.1. Let $\Omega_{1}, \ldots, \Omega_{N}$ be measurable subsets of $\widehat{G}$, and let $\psi_{j}=\breve{\mathbf{1}}_{\Omega_{j}}$ for each $j=1, \ldots, N$. We say that $\left\{\Omega_{1}, \ldots, \Omega_{N}\right\}$ is a wavelet collection of sets if $\Psi=\left\{\psi_{1}, \ldots, \psi_{N}\right\}$ is a set of wavelet generators for $L^{2}(G)$.

If $N=1$, then $\Omega=\Omega_{1}$ is a wavelet set. 
Based on a general formulation by Dai and Larson [19] (with an early version available in 1993) and by Dai, Larson, and Speegle [20], wavelet sets in $\widehat{\mathbb{R}}^{d}$ were constructed by Soardi and Weiland [56] and by Leon and one of the authors $[10,11]$ using the notion of $\tau$-congruence, which gives an equivalence relation between subsets of $\widehat{\mathbb{R}}^{d}$. We now formulate an analogous equivalence relation for LCAGs $G$ with compact open subgroups $H$.

Definition 3.3. Let $G$ be a LCAG with compact open subgroup $H \subseteq G$, let $\mathcal{D} \subseteq \widehat{G}$ be a choice of coset representatives in $\widehat{G}$ for $\widehat{H}=\widehat{G} / H^{\perp}$, and let $V$ and $V^{\prime}$ be subsets of $\widehat{G}$. We say $V$ is $(\tau, \mathcal{D})$-congruent to $V^{\prime}$ if there is a finite or countable indexing set $I \subseteq \mathbb{Z}$ as well as partitions $\left\{V_{n}: n \in I\right\}$ and $\left\{V_{n}^{\prime}: n \in I\right\}$ of $V$ and $V^{\prime}$, respectively, into measurable subsets, and sequences $\left\{\sigma_{n}\right\}_{n \in I},\left\{\sigma_{n}^{\prime}\right\}_{n \in I} \subseteq \mathcal{D}$ such that

$$
\forall n \in I, \quad V_{n} \subseteq \sigma_{n}+H^{\perp} \quad \text { and } \quad V_{n}=V_{n}^{\prime}-\sigma_{n}^{\prime}+\sigma_{n} .
$$

If $V_{n}=V_{n}^{\prime}-\sigma_{n}^{\prime}+\sigma_{n}$, then the condition that $V_{n} \subseteq \sigma_{n}+H^{\perp}$ is equivalent to $V_{n}^{\prime} \subseteq \sigma_{n}^{\prime}+H^{\perp}$. Thus, in the case $V^{\prime}=H^{\perp}$, we take $\sigma_{n}^{\prime}=\sigma_{0}^{\prime}$ for all $n$, where $\sigma_{0}^{\prime}$ is the unique element of $\mathcal{D} \cap H^{\perp}$. Note that the notion of $(\tau, \mathcal{D})$-congruence depends crucially on the choice of coset representatives $\mathcal{D}$. Clearly, $(\tau, \mathcal{D})$-congruence is an equivalence relation, and it preserves Haar measure.

Remark 3.4. (a) Definition 3.3 is related to bijective restrictions of the canonical surjection defined in (1.2); see an analysis of this relation in [8, Section 3] in the context of Kluvánek's sampling theorem (1965) for LCAGs, cf., Remark 1.1. Kluvánek's sampling formula for a signal $f$ quantitatively relates the sampling rate with the measure of the subsets of a given bandwidth corresponding to the frequency content of $f$.

(b) Congruence criteria were introduced by Albert Cohen (1990) to check the orthonormality of scaling functions of MRAs defined by infinite products of dilations of a conjugate mirror filter, e.g., [23], p. 182-186. The same notion of congruence also plays a fundamental role in work on self-similar tilings by Gröchenig, et al., e.g., [28, 27, 37, 38]. We are dealing with (possibly infinite) sets $\mathcal{D} \subseteq \widehat{G}$ of coset representatives in $\widehat{G}$ for $\widehat{G} / H^{\perp}$, whereas the previous references deal with coset representatives, so called digits, of each coset of $A\left(\mathbb{Z}^{d}\right)$ in $\mathbb{Z}^{d}$. In this latter case, $A: \mathbb{R}^{d} \longrightarrow \mathbb{R}^{d}$ is an expansive matrix, and a goal is to determine those $A$ for which a set of digits exists allowing for the construction of MRAs with 0-1 valued scaling functions.

\subsection{Characterization of wavelet collections of sets}

Let $X$ be a measurable subset of $\widehat{G}$, and let $\left\{X_{n}: n \in \mathbb{Z}\right\}$ be a countable set of measurable subsets of $\widehat{G}$. We say that $\left\{X_{n}\right\}$ tiles $X$ up to sets of measure zero if both

$$
v\left(X \backslash\left[\bigcup_{n \in \mathbb{Z}} X_{n}\right]\right)=0
$$

and

$$
\forall m, n \in \mathbb{Z}, m \neq n, \quad v\left(X_{m} \cap X_{n}\right)=0 .
$$

Similarly, we say that two sets $X, Y \subseteq \widehat{G}$ are $(\tau, \mathcal{D})$-congruent up to sets of measure zero if there are $(\tau, \mathcal{D})$-congruent sets $X^{\prime}, Y^{\prime} \subseteq \widehat{G}$ such that

$$
v\left(X \backslash X^{\prime}\right)=v\left(X^{\prime} \backslash X\right)=v\left(Y \backslash Y^{\prime}\right)=v\left(Y^{\prime} \backslash Y\right)=0 .
$$

We are now prepared to state and prove our first main result, which gives a necessary and sufficient condition for a set $\Omega \subseteq \widehat{G}$ to be a wavelet set. 
Theorem 3.5. Let $G$ be a $L C A G$ with compact open subgroup $H \subseteq G$, let $\mathcal{D} \subseteq \widehat{G}$ be a choice of coset representatives in $\widehat{G}$ for $\widehat{H}=\widehat{G} / H^{\perp}$, and let $\mathcal{A} \subseteq$ Aut $G$ be a countable nonempty subset of Aut $G$. Consider a finite sequence $\left\{\Omega_{1}, \ldots, \Omega_{N}\right\}$ of measurable subsets of $\widehat{G} .\left\{\Omega_{1}, \ldots, \Omega_{N}\right\}$ is a wavelet collection of sets if and only if both of the following conditions hold:

(a) $\left\{A^{*} \Omega_{j}: A \in \mathcal{A}, j=1, \ldots, N\right\}$ tiles $\widehat{G}$ up to sets of measure zero, and

(b) $\forall j=1, \ldots, N, \Omega_{j}$ is $(\tau, \mathcal{D})$-congruent to $H^{\perp}$ up to sets of measure zero.

In that case, $\widehat{G}$ is $\sigma$-compact, each $v\left(\Omega_{j}\right)=1$, and each $\mathbf{1}_{\Omega_{j}} \in L^{2}(\widehat{G})$.

Proof. Let $\psi_{j}=\check{\mathbf{1}}_{\Omega_{j}}$, and recall that $\psi_{j, A,[s]}=\delta_{A} \tau_{[s], \mathcal{D}} \psi_{j}$. Recall also that $\sigma_{0}^{\prime}$ is the unique element of $\mathcal{D} \cap H^{\perp}$.

(i) We first verify that property (b) implies that each $v\left(\Omega_{j}\right)=1$. Let $I_{j} \subseteq \mathbb{Z}$ be the index set for the congruence from Definition 3.3, and let $\left\{V_{j, n}: n \in I_{j}\right\}$ be the corresponding partition of $\Omega_{j}$. Since $\Omega_{j}$ is $(\tau, \mathcal{D})$-congruent to $H^{\perp}$, we have

$$
v\left(\Omega_{j}\right)=\sum_{n \in I_{j}} v\left(V_{j, n}\right)=\sum_{n \in I_{j}} v\left(V_{j, n}+\sigma_{n}^{\prime}-\sigma_{n}\right)=\sum_{n \in I_{j}} v\left(V_{j, n}^{\prime}\right)=v\left(H^{\perp}\right)=1,
$$

where $\left\{V_{j, n}^{\prime}: n \in I_{j}\right\}$ is a partition of $H^{\perp}$, and $\sigma_{n}^{\prime}=\sigma_{0}^{\prime}$ for all $n \in I_{j}$. In particular, when property (b) is assumed, we have $\mathbf{1}_{\Omega_{j}} \in L^{2}(\widehat{G})$. Moreover, $\left\|\mathbf{1}_{\Omega_{j}}\right\|=1$, so that $\left\|\psi_{j}\right\|_{2}=1$.

Conversely, if $\left\{\Omega_{1}, \ldots, \Omega_{N}\right\}$ is a wavelet collection of sets, then

$$
1=\left\|\psi_{j}\right\|_{2}=\left\|\mathbf{1}_{\Omega_{j}}\right\|_{2}=v\left(\Omega_{j}\right) .
$$

We shall also need the fact that property (a) implies that $\Omega_{1}, \ldots, \Omega_{N}$ are pairwise disjoint up to sets of measure zero. To see this, pick any $A \in \mathcal{A}$ and observe that for $j \neq k$,

$$
v\left(\Omega_{j} \cap \Omega_{k}\right)=|A|^{-1} v\left(A^{*}\left(\Omega_{j} \cap \Omega_{k}\right)\right)=|A|^{-1} v\left(\left(A^{*} \Omega_{j}\right) \cap\left(A^{*} \Omega_{k}\right)\right)=0
$$

by property (a).

(ii) We now prove that properties (a) and (b) imply that $\left\{\psi_{j, A,[s]}: 1 \leq j \leq N, A \in \mathcal{A},[s] \in\right.$ $G / H\}$ is an orthonormal set.

First observe that $\left\|\psi_{j, A,[s]}\right\|_{2}=1$ for any $A \in \mathcal{A}$ and $[s] \in G / H$. Indeed, we compute:

$$
\begin{aligned}
\left\|\psi_{j, A,[s]}\right\|_{2}^{2} & =\int_{\widehat{G}}\left|\widehat{\psi_{j, A,[s]}}(\gamma)\right|^{2} d \gamma=|A|^{-1} \int_{\widehat{G}}\left|\widehat{\psi_{j}}\left(\left(A^{*}\right)^{-1} \gamma\right)\right|^{2} d \gamma \\
& =\int_{\widehat{G}}\left|\widehat{\psi_{j}}(\beta)\right|^{2} d \beta=\int_{\Omega_{j}} d \beta=v\left(\Omega_{j}\right)=1,
\end{aligned}
$$

by the change of variables $\beta=\left(A^{*}\right)^{-1} \gamma$. Furthermore, if $(j, B,[t])$ and $(k, C,[u])$ are distinct triples in $\{1, \ldots, N\} \times \mathcal{A} \times(G / H)$, then $\left\langle\psi_{j, B,[t]}, \psi_{k, C,[u]}\right\rangle=0$. To see this, consider the cases $(j, B) \neq(k, C)$ and $(j, B)=(k, C)$.

If $(j, B) \neq(k, C)$, then we compute

$\left\langle\psi_{j, B,[t]}, \psi_{k, C,[u]}\right\rangle$

$$
\begin{aligned}
& =(|B||C|)^{-1 / 2} \int_{\widehat{G}} \widehat{\psi_{j}}\left(\left(B^{*}\right)^{-1} \gamma\right) \overline{\widehat{\psi_{k}}\left(\left(C^{*}\right)^{-1} \gamma\right)\left(t, \eta\left(\left(B^{*}\right)^{-1} \gamma\right)\right)}\left(u, \eta\left(\left(C^{*}\right)^{-1} \gamma\right)\right) d \gamma \\
& =(|B \| C|)^{-1 / 2} \int_{\widehat{G}} \mathbf{1}_{B^{*} \Omega_{j}}(\gamma) \mathbf{1}_{C^{*} \Omega_{k}}(\gamma) \overline{\left(t, \eta\left(\left(B^{*}\right)^{-1} \gamma\right)\right)}\left(u, \eta\left(\left(C^{*}\right)^{-1} \gamma\right)\right) d \gamma=0,
\end{aligned}
$$


because the intersection $\left(B^{*} \Omega_{j}\right) \cap\left(C^{*} \Omega_{k}\right)$ has measure zero, by property (a).

If $(j, B)=(k, C)$ but $[t] \neq[u]$, recall that, by property $(\mathrm{b}), \Omega_{j}$ is $(\tau, \mathcal{D})$-congruent to $H^{\perp}$ up to sets of measure zero. We may therefore write $\Omega_{j}$ (up to sets of measure zero) as the disjoint union of sets $V_{j, n}$, where $n$ ranges over a finite or countable index set $I_{j}$, and where $H^{\perp}$ is the disjoint union of the sets $V_{j, n}^{\prime}=V_{j, n}-\sigma_{j, n}+\sigma_{0}^{\prime}$, where each $\sigma_{j, n} \in \mathcal{D}$. Therefore,

$$
\begin{aligned}
\left\langle\psi_{j, B,[t]}, \psi_{j, B,[u]}\right\rangle & =\int_{\Omega_{j}}(u-t, \eta(\gamma)) d \gamma \\
& =\sum_{n \in I_{j}} \int_{V_{j, n}}(u-t, \eta(\gamma)) d \gamma=\sum_{n \in I_{j}} \int_{V_{j, n}^{\prime}-\sigma_{0}^{\prime}+\sigma_{n}}(u-t, \eta(\gamma)) d \gamma \\
& =\sum_{n \in I_{j}} \int_{V_{j, n}^{\prime}}\left(u-t, \eta\left(\lambda-\sigma_{0}^{\prime}+\sigma_{n}\right)\right) d \lambda=\sum_{n \in I_{j}} \int_{V_{j, n}^{\prime}}\left(u-t, \lambda-\sigma_{0}^{\prime}\right) d \lambda \\
& =\overline{\left(u-t, \sigma_{0}^{\prime}\right)} \int_{H^{\perp}}(u-t, \lambda) d \lambda
\end{aligned}
$$

where the second, third, and sixth equalities follow from the definition of $(\tau, \mathcal{D})$-congruence, and the fourth is the change of variables $\lambda=\gamma-\sigma_{n}+\sigma_{0}^{\prime}$. The fifth follows from the definition of $\eta$, since $\lambda-\sigma_{0}^{\prime} \in H^{\perp}$, so that $\theta\left(\lambda-\sigma_{0}^{\prime}+\sigma_{n}\right)=\sigma_{n}$, and hence

$$
\eta\left(\lambda-\sigma_{0}^{\prime}+\sigma_{n}\right)=\left(\lambda-\sigma_{0}^{\prime}+\sigma_{n}\right)-\sigma_{n}=\lambda-\sigma_{0}^{\prime} .
$$

Finally, by the following well-known proof, (3.2) vanishes because $[t] \neq[u]$, i.e., because $x=u-t \notin H^{\perp}$ :

$$
\int_{H^{\perp}}(x, \lambda) d v_{H^{\perp}}(\lambda)=\left(x, \gamma_{0}\right) \int_{H^{\perp}}\left(x, \lambda-\gamma_{0}\right) d v_{H^{\perp}}(\lambda)=\left(x, \gamma_{0}\right) \int_{H^{\perp}}(x, \lambda) d v_{H^{\perp}}(\lambda),
$$

where we have used the translation invariance of $v_{H^{\perp}}$ and where $\gamma_{0} \in H^{\perp}$ was chosen so that $\left(x, \gamma_{0}\right) \neq 1$.

Thus, $\left\{\psi_{j, A,[s]}: 1 \leq j \leq N, A \in \mathcal{A},[s] \in G / H\right\}$ is an orthonormal set.

(iii) Next, we prove that properties (a) and (b) are sufficient for $\left\{\Omega_{1}, \ldots, \Omega_{N}\right\}$ to be a wavelet collection of sets. The substantive part of the proof is concerned with proving the following formula:

$$
\forall f \in L^{2}(G), \quad \sum_{\substack{1 \leq j \leq N \\ A \in \mathcal{A} \\[s] \in G / H}}\left|\left\langle f, \psi_{j, A,[s]}\right\rangle\right|^{2}=\|f\|_{2}^{2} .
$$

This fact, combined with the orthonormality, proves that $\left\{\psi_{j, A,[s]}: 1 \leq j \leq N, A \in \mathcal{A},[s] \in\right.$ $G / H\}$ is an orthonormal basis for $L^{2}(G)$.

Now to the proof of (3.3). By the orthonormality, the left side of (3.3) is bounded by $\|f\|_{2}^{2}$ when the sum is over any finite set of indices (Bessel's inequality). Thus,

$$
\forall f \in L^{2}(G), \quad \operatorname{card}\left\{\psi_{j, A,[s]}:\left\langle f, \psi_{j, A,[s]}\right\rangle \neq 0\right\} \leq \aleph_{o},
$$

and so the left side of (3.3) is finite, and we shall evaluate it. By Plancherel's theorem and (2.8), 
we have

$$
\begin{aligned}
\sum_{\substack{1 \leq j \leq N \\
A \in \mathcal{A} \\
[s] \in G / H}}\left|\left\langle f, \psi_{j, A,[s]}\right\rangle\right|^{2} & =\sum_{\substack{1 \leq j \leq N \\
A \in \mathcal{A} \\
[s] \in G / H}}\left|\left\langle\hat{f}, \widehat{\psi_{j, A,[s}}\right\rangle\right|^{2} \\
& =\sum_{\substack{1 \leq j \leq N \\
\text { A } \\
[s] \in G / H}}|A|^{-1}\left|\int_{\widehat{G}} \hat{f}(\gamma) \overline{\widehat{\psi_{j}}\left(\left(A^{*}\right)^{-1} \gamma\right)}\left(s, \eta\left(\left(A^{*}\right)^{-1} \gamma\right)\right) d \gamma\right|^{2} \\
& =\sum_{\substack{1 \leq j \leq N \\
A \in \mathcal{A} \\
[s] \in G / H}}|A|\left|\int_{\widehat{G}} \hat{f}\left(A^{*} \beta\right) \widehat{\widehat{\psi_{j}}(\beta)}(s, \eta(\beta)) d \beta\right|^{2}
\end{aligned}
$$

where we have substituted $\beta=\left(A^{*}\right)^{-1} \gamma$, and hence $d \beta=|A|^{-1} d \gamma$, i.e., (1.1).

By property (b), $\Omega_{j}$ is ( $\tau, \mathcal{D}$ )-congruent to $H^{\perp}$ up to sets of measure zero. Recalling the notation $I_{j}, V_{j, n}^{\prime}, V_{j, n}$, and $\sigma_{j, n}$ from the discussion preceding (3.2), the right side of (3.4) becomes

$$
\begin{aligned}
& \sum_{\substack{1 \leq j \leq N \\
A \in \mathcal{A} \\
[s] \in G / H}}|A|\left|\int_{\Omega_{j}} \hat{f}\left(A^{*} \beta\right)(s, \eta(\beta)) d \beta\right|^{2} \\
& =\sum_{\substack{1 \leq j \leq N \\
\text { a } \\
[s] \in G / H}}|A|\left|\sum_{n \in I_{j}}\left[\int_{V_{j, n}} \hat{f}\left(A^{*} \beta\right)(s, \eta(\beta)) d \beta\right]\right|^{2} \\
& =\sum_{\substack{1 \leq j \leq N \\
A \in \mathcal{A} \\
[s] \in G / H}}|A|\left|\sum_{n \in I_{j}}\left[\int_{V_{j, n}^{\prime}-\sigma_{0}^{\prime}} \hat{f}\left(A^{*}\left(\alpha+\sigma_{j, n}\right)\right)\left(s, \eta\left(\alpha+\sigma_{j, n}\right)\right) d \alpha\right]\right|^{2} \\
& =\sum_{\substack{1 \leq j \leq N \\
A \in \mathcal{A} \\
[s] \in G / H}}|A|\left|\sum_{n \in I_{j}}\left[\int_{\widehat{G}} \mathbf{1}_{V_{j, n}^{\prime}}\left(\alpha+\sigma_{0}^{\prime}\right) \hat{f}\left(A^{*}\left(\alpha+\sigma_{j, n}\right)\right)\left(s, \eta\left(\alpha+\sigma_{j, n}\right)\right) d \alpha\right]\right|^{2},
\end{aligned}
$$

where we have substituted $\alpha=\beta-\sigma_{j, n}$. Since $\alpha \in H^{\perp}-\sigma_{0}^{\prime}=H^{\perp}$, we have

$$
\eta\left(\alpha+\sigma_{j, n}\right)=\left(\alpha+\sigma_{j, n}\right)-\theta\left(\alpha+\sigma_{j, n}\right)=\left(\alpha+\sigma_{j, n}\right)-\sigma_{j, n}=\alpha .
$$

Furthermore, we can exchange the inner summation and integral signs in the last term of (3.5). In fact, since $\left\{V_{j, n}^{\prime}\right\}$ is a tiling of $H^{\perp}$ and $K_{n}=V_{j, n}^{\prime}-\sigma_{0}^{\prime} \subseteq H^{\perp}$, then $\left\{K_{n}\right\}$ is a tiling of $H^{\perp}$; and, hence, denoting the integrand by $F_{n}$ (noting it vanishes off $K_{n}$ ), and writing $F=\sum F_{n}$, we see that $F_{n}, F \in L^{2}\left(H^{\perp}\right) \subseteq L^{1}\left(H^{\perp}\right)$, that $\bigcup K_{n}=H^{\perp}$, and that

$$
\sum_{n \in I_{j}} \int_{\widehat{G}} F_{n}(\alpha) d \alpha=\sum_{n \in I_{j}} \int_{K_{n}} F(\alpha) d \alpha=\int_{H^{\perp}} F(\alpha) d \alpha
$$


Thus, the right side of (3.5) becomes

$$
\sum_{\substack{1 \leq j \leq N \\ A \in \mathcal{A}}}|A| \sum_{[s] \in G / H}\left|\int_{H^{\perp}}\left[\sum_{n \in I_{j}} \mathbf{1}_{V_{j, n}^{\prime}}\left(\alpha+\sigma_{0}^{\prime}\right) \hat{f}\left(A^{*}\left(\alpha+\sigma_{j, n}\right)\right)\right](s, \alpha) d \alpha\right|^{2} .
$$

Let $F_{j, A}(\alpha)=\sum_{n \in I_{j}} \mathbf{1}_{V_{j, n}^{\prime}}\left(\alpha+\sigma_{0}^{\prime}\right) \hat{f}\left(A^{*}\left(\alpha+\sigma_{j, n}\right)\right)$ on $H^{\perp} \subseteq \widehat{G}$. In particular, supp $F_{j, A} \subseteq$ $H^{\perp}$. We consider $F_{j, A}$ as an element of $L^{2}\left(H^{\perp}\right)$, where the Haar measure $v_{H^{\perp}}$ may be considered the restriction of $v=v_{\widehat{G}}$ to the compact group $H^{\perp} \subseteq \widehat{G}$ with the scaling $v_{H^{\perp}}\left(H^{\perp}\right)=v\left(H^{\perp}\right)=1$, as noted in Section 1.3. As such, we have

$$
\int_{\widehat{G}} F_{j, A}(\alpha) d v(\alpha)=\int_{H^{\perp}} F_{j, A}(\alpha) d v_{H^{\perp}}(\alpha) .
$$

Hence, since $G / H$ is the discrete dual group of $H^{\perp}$, we may apply Plancherel's theorem for Fourier series and obtain

$$
\sum_{[s] \in G / H}\left|\int_{H^{\perp}} F_{j, A}(\alpha)([s], \alpha) d v(\alpha)\right|^{2}=\int_{H^{\perp}}\left|F_{j, A}(\alpha)\right|^{2} d v(\alpha) .
$$

Thus, because $([s], \alpha)=(s, \alpha)$ for $[s] \in G / H$ and $\alpha \in H^{\perp}$, (3.6) becomes

$$
\sum_{\substack{1 \leq j \leq N \\ A \in \mathcal{A}}}|A| \int_{H^{\perp}}\left|\sum_{n \in I_{j}} \mathbf{1}_{V_{j, n}^{\prime}}\left(\alpha+\sigma_{0}^{\prime}\right) \hat{f}\left(A^{*}\left(\alpha+\sigma_{j, n}\right)\right)\right|^{2} d \alpha
$$

which, in turn, is

$$
\sum_{\substack{1 \leq j \leq N \\ A \in \mathcal{A}}}|A| \int_{H^{\perp}}\left[\sum_{n \in I_{j}}\left|\hat{f}\left(A^{*}\left(\alpha+\sigma_{j, n}\right)\right)\right|^{2} \mathbf{1}_{V_{j, n}^{\prime}}\left(\alpha+\sigma_{0}^{\prime}\right)\right] d \alpha,
$$

where (3.7) is a consequence of the fact that, for $j$ fixed, the sets $V_{j, n}^{\prime}$ are pairwise disjoint, so that for any given $\alpha$ at most one term in the sum over $n$ is nonzero. We can now interchange the inner summation and integral as before. Hence, (3.7) becomes

$$
\begin{aligned}
& \sum_{\substack{1 \leq j \leq N \\
A \in \mathcal{A}}}|A| \sum_{n \in I_{j}}\left[\int_{H^{\perp}}\left|\hat{f}\left(A^{*}\left(\alpha+\sigma_{j, n}\right)\right)\right|^{2} \mathbf{1}_{V_{j, n}^{\prime}}\left(\alpha+\sigma_{0}^{\prime}\right) d \alpha\right] \\
& =\sum_{\substack{1 \leq j \leq N \\
A \in \mathcal{A}}}|A| \sum_{n \in I_{j}}\left[\int_{V_{j, n}^{\prime}-\sigma_{0}^{\prime}}\left|\hat{f}\left(A^{*}\left(\alpha+\sigma_{j, n}\right)\right)\right|^{2} d \alpha\right] \\
& =\sum_{\substack{1 \leq j \leq N \\
A \in \mathcal{A}}}|A| \sum_{n \in I_{j}}\left[\int_{V_{j, n}}\left|\hat{f}\left(A^{*}(\beta)\right)\right|^{2} d \beta\right] \\
& =\sum_{\substack{1 \leq j \leq N \\
A \in \mathcal{A}}}|A| \int_{\Omega_{j}}\left|\hat{f}\left(A^{*}(\beta)\right)\right|^{2} d \beta \\
& =\sum_{\substack{1 \leq j \leq N \\
A \in \mathcal{A}}} \int_{A^{*} \Omega_{j}}|\hat{f}(\gamma)|^{2} d \gamma .
\end{aligned}
$$


However, $\left\{A^{*} \Omega_{j}\right\}$ tiles $\widehat{G}$ up to sets of measure zero. Hence, the right side of (3.8) is

$$
\int_{\widehat{G}}|\hat{f}(\gamma)|^{2} d \gamma=\|\hat{f}\|_{2}^{2}=\|f\|_{2}^{2},
$$

and so formula (3.3) is proved.

(iv) Let $\left\{\Omega_{1}, \ldots, \Omega_{N}\right\}$ be a wavelet collection of sets. We now prove that $\widehat{G}$ is $\sigma$-compact. Note that for any $A \in \mathcal{A}$ and $1 \leq j \leq N$,

$$
v\left(A^{*} \Omega_{j}\right)=|A| v\left(\Omega_{j}\right)=|A|<\infty .
$$

Thus, there are at most countably many $\sigma \in \mathcal{D}$ such that $\nu\left(\left(\sigma+H^{\perp}\right) \cap\left(A^{*} \Omega_{j}\right)\right)>0$. Write $X=\bigcup_{A \in \mathcal{A}, 1 \leq j \leq N} A^{*} \Omega_{j}$, which is a countable union; hence, there are at most countably many $\sigma \in \mathcal{D}$ such that $v\left(\left(\sigma+H^{\perp}\right) \cap X\right)>0$.

Suppose $\mathcal{D}$ were uncountable; then there is some $\sigma \in \mathcal{D}$ such that $v\left(\left(\sigma+H^{\perp}\right) \cap X\right)=0$. Let $F=\mathbf{1}_{\sigma+H^{\perp}} \in L^{2}(\widehat{G})$. Then for any $A \in \mathcal{A}, 1 \leq j \leq N$, and $[s] \in G / H$, we have $\left\langle F, \widehat{\psi_{j, A,[s]}}\right\rangle=0$, because $\widehat{\psi_{j, A,[s]}}$ vanishes off of $X$. Therefore,

$$
1=\|F\|_{2}^{2}=\sum_{j, A,[s]}\left|\left\langle F, \widehat{\psi_{j, A,[s]}}\right\rangle\right|^{2}=0 .
$$

By this contradiction, it follows that $\mathcal{D}$ is countable. Thus, $\widehat{G}=\bigcup_{\sigma \in \mathcal{D}}\left(\sigma+H^{\perp}\right)$ is a countable union of compact sets, as desired.

(v) Finally, we prove that properties (a) and (b) are necessary conditions for $\left\{\psi_{j}\right\}$ to be a set of wavelet generators.

First we show that $\left\{A^{*} \Omega_{j}\right\}$ tiles $\widehat{G}$ up to set of measure zero [property (a)]. If $(j, B),(k, C) \in$ $\{1, \ldots, N\} \times \mathcal{A}$ are distinct pairs, then $\left\langle\psi_{j, B,[0]}, \psi_{j, C,[0]}\right\rangle=0$ by orthonormality. Thus,

$$
v\left(B^{*} \Omega_{j} \cap C^{*} \Omega_{k}\right)=\left\langle\mathbf{1}_{B^{*} \Omega_{j}}, \mathbf{1}_{C^{*} \Omega_{k}}\right\rangle=\left\langle\psi_{j, B,[0]}, \psi_{k, C,[0]}\right\rangle=0 .
$$

If $\alpha \in Y=\left(\widehat{G} \backslash\left[\bigcup_{A \in \mathcal{A}, 1 \leq j \leq N} A^{*} \Omega_{j}\right]\right)$, then let $Y_{\alpha}=Y \cap\left(\alpha+H^{\perp}\right)$. Clearly $\mathbf{1}_{Y_{\alpha}} \in L^{2}(\widehat{G})$, and the orthonormality of $\left\{\psi_{j, A,[s]}\right\}$ ensures that $\operatorname{card}\left\{(j, A,[s]):\left\langle\mathbf{1}_{Y_{\alpha}}, \psi_{j, A,[s]}\right\rangle \neq 0\right\} \leq \aleph_{0}$. Since $\left\{\psi_{j, A,[s]}\right\}$ is an orthonormal basis for $L^{2}(G)$, we have

$$
\begin{aligned}
& v\left(Y_{\alpha}\right)=\left\|\mathbf{1}_{Y_{\alpha}}\right\|_{2}^{2}=\sum_{\substack{1 \leq j \leq N \\
\text { A } \in \mathcal{A} \\
[s] \in G / H}}\left|\left\langle\mathbf{1}_{Y_{\alpha}}, \widehat{\psi_{j, A,[s]}}\right)\right|^{2} \\
& =\sum_{\substack{1 \leq j \leq N \\
\text { a } \\
[s] \in G / H}}|A|^{-1}\left|\int_{\widehat{G}} \mathbf{1}_{Y_{\alpha}}(\gamma) \widehat{\bar{\psi}_{j}}\left(\left(A^{*}\right)^{-1} \gamma\right)\left(s, \eta\left(\left(A^{*}\right)^{-1} \gamma\right)\right) d \gamma\right|^{2} \\
& =\sum_{\substack{1 \leq j \leq N \\
A \in \mathcal{A} \\
[s] \in G / H}}|A|^{-1}\left|\int_{Y_{\alpha} \cap A^{*} \Omega_{j}}\left(s, \eta\left(\left(A^{*}\right)^{-1} \gamma\right)\right) d \gamma\right|^{2}=0,
\end{aligned}
$$

because $Y_{\alpha} \cap A^{*} \Omega_{j}=\emptyset$. We have covered $Y$ by open sets $\alpha+H^{\perp}$ such that each intersection $Y \cap\left(\alpha+H^{\perp}\right)$ has measure zero; thus, $v(Y)=0$, by the $\sigma$-compactness of $\widehat{G}$. 
Second, we show that each $\Omega_{j}$ is $(\tau, \mathcal{D})$-congruent to $H^{\perp}$ [property (b)]. Since $v\left(\Omega_{j}\right)=$ $1<\infty$, there are at most countably many cosets $\sigma+H^{\perp}$ such that $\Omega_{j} \cap\left(\sigma+H^{\perp}\right)$ has positive measure. (In fact, $\widehat{G} / H^{\perp}$ is countable because $\widehat{G}$ is $\sigma$-compact.) Let $\left\{\sigma_{j, n}: n \in I_{j}\right\}$ be the set of all such $\sigma \in \mathcal{D}$, where $I_{j} \subseteq \mathbb{Z}$ is some finite or countable indexing set. For each $n \in I_{j}$, let $V_{j, n}=\Omega_{j} \cap\left(\sigma_{j, n}+H^{\perp}\right)$ and let $V_{j, n}^{\prime}=V_{j, n}-\sigma_{j, n}+\sigma_{0}^{\prime}$, where $\sigma_{0}^{\prime}$ is the unique element of $\mathcal{D} \cap H^{\perp}$.

Clearly, $\left\{V_{j, n}\right\}$ is a partition of $\Omega_{j}$, by the definitions of $\sigma_{j, n}$ and $V_{j, n}$.

It remains to prove that $\left\{V_{j, n}^{\prime}\right\}$ is a partition of $H^{\perp}$. The proof requires our hypothesis of orthonormality, as follows. Let $j \in\{1, \ldots, N\}$ and $A \in \mathcal{A}$ be fixed, and compute, for any $[s] \in G / H$, that

$$
\begin{aligned}
\delta([s]) & =\left\langle\psi_{j, A,[0],}, \psi_{j, A,[s]}\right\rangle \\
& =|A|^{-1} \int_{\widehat{G}}\left|\widehat{\psi}_{j}\left(\left(A^{*}\right)^{-1} \gamma\right)\right|^{2} \overline{\left(s, \eta\left(\left(A^{*}\right)^{-1} \gamma\right)\right)} d \gamma \\
& =\int_{\Omega_{j}} \overline{(s, \eta(\gamma))} d \gamma=\sum_{n \in I_{j}} \int_{V_{j, n}} \overline{(s, \eta(\gamma))} d \gamma \\
& =\sum_{n \in I_{j}} \int_{V_{j, n}-\sigma_{j, n}} \overline{\left(s, \eta\left(\sigma_{j, n}+\beta\right)\right)} d \beta \\
& =\sum_{n \in I_{j}} \int_{V_{j, n}-\sigma_{j, n}} \overline{(s, \beta)} d \beta,
\end{aligned}
$$

where $\delta([s])=0$ if $[s] \neq[0]$ and $\delta([s])=1$ if $[s]=[0]$.

Since $j$ is fixed, let

$$
F=\sum_{n \in I_{j}} \mathbf{1}_{V_{j, n}-\sigma_{j, n}} \quad \text { and } \quad F_{M}=\sum_{n \in I_{j, M}} \mathbf{1}_{V_{j, n}-\sigma_{j, n}}
$$

where the finite sets $I_{j, M}$ increase to $I_{j}$ as $M \rightarrow \infty$. We shall show that $F \in L^{1}\left(H^{\perp}\right)$, noting that $\operatorname{supp} F_{M} \subseteq \operatorname{supp} F \subseteq H^{\perp}$. First,

$$
\int_{H^{\perp}} F_{M}(\gamma) d \gamma=\sum_{n \in I_{j, M}} \int_{H^{\perp}} \mathbf{1}_{V_{j, n}-\sigma_{j, n}}(\gamma) d \gamma=\sum_{n \in I_{j, M}} v\left(V_{j, n}\right) ;
$$

and it is clear that

$$
0 \leq F_{M} \leq F \quad \text { and } \quad \lim _{M \rightarrow \infty} F_{M}=F \quad v-\text { a.e. }
$$

The Beppo Levi theorem applies by (3.11), and so, using (3.10),

$$
\int_{H^{\perp}} F(\gamma) d \gamma=\lim _{M \rightarrow \infty} \int_{H^{\perp}} F_{M}(\gamma) d \gamma=\lim _{M \rightarrow \infty} \sum_{n \in I_{j, M}} v\left(V_{j, n}\right)=1 .
$$

Thus, $F \in L^{1}\left(H^{\perp}\right)$ since $F \geq 0$. Hence, we can apply the Lebesgue dominated convergence theorem to the sequence $\left\{F_{M}(\cdot) \overline{(s, \cdot)}\right\}$, which converges $v$-a.e. to $F(\cdot) \overline{(s, \cdot)}$ and which is bounded in absolute value by $F \in L^{1}\left(H^{\perp}\right)$. Therefore, (3.9) becomes

$$
\delta([s])=\lim _{M \rightarrow \infty} \sum_{n \in I_{j, M}} \int_{V_{j, n}-\sigma_{j, n}} \overline{(s, \beta)} d \beta=\lim _{M \rightarrow \infty} \int_{H^{\perp}} F_{M}(\beta) \overline{(s, \beta)} d \beta=\int_{H^{\perp}} F(\beta) \overline{(s, \beta)} d \beta,
$$


where we can once again consider $v_{H^{\perp}}$ as the restriction of $v$ to $H^{\perp}$ since $H^{\perp}$ is open in $\widehat{G}$.

By the $L^{1}$-uniqueness theorem for Fourier series, we must have $F=1$ on $H^{\perp}$. Moreover, because $F=\sum_{n \in I_{j}} \mathbf{1}_{V_{j, n}-\sigma_{j, n}}$, it follows that $\left\{V_{j, n}-\sigma_{j, n}\right\}$ is a partition of $H^{\perp}$. Hence, $\left\{V_{j, n}^{\prime}-\sigma_{0}^{\prime}\right\}$, and therefore $\left\{V_{j, n}^{\prime}\right\}$, are partitions of $H^{\perp}$.

Remark 3.6. (a) The content of part (iv) is the assertion that either the necessary or the sufficient conditions in Theorem 3.5 imply that $\widehat{G}$ is $\sigma$-compact. The proof we give shows this implication when assuming that $\left\{\Omega_{1}, \ldots, \Omega_{N}\right\}$ is a wavelet collection of sets; the fact that the sufficient conditions also imply $\sigma$-compactness then follows from part (iii). However, it is easy to check that condition (a) of Theorem 3.5 implies that $\widehat{G}$ is $\sigma$-compact directly, without appealing to (iii).

(b) The proof that conditions (a) and (b) are sufficient for a wavelet collection of sets does not a priori require $\widehat{G}$ to be $\sigma$-compact, even though condition (a) easily implies $\sigma$-compactness, and condition (b) implies that each $v\left(\Omega_{j}\right)=1$. On the other hand, the $\sigma$-compactness is required in the proof, in part (v), that the conditions are necessary.

(c) Part (iv) shows that $\widehat{G}$ is $\sigma$-compact by showing that $\mathcal{D}$, and hence $\widehat{G} / H^{\perp}$, is countable. In fact, these two conditions are equivalent; the proof is elementary.

(d) Recall that a LCAG $G$ is metrizable if and only if $\widehat{G}$ is $\sigma$-compact.

(e) Also recall, as noted before Lemma 2.6, that if there is an expansive automorphism $A \in$ Aut $G$, then $\widehat{G}$ is $\sigma$-compact.

\section{The construction of wavelet sets}

\subsection{The algorithm}

We are now prepared to construct wavelet collections of sets. As before, $G$ is a LCAG with compact open subgroup $H$, and $\mathcal{D}$ is a choice of coset representatives in $\widehat{G}$ for the quotient $\widehat{G} / H^{\perp}$. Let $A_{1} \in$ Aut $G$ be expansive with respect to $H$, and let $\mathcal{A}=\left\{A_{1}^{n}: n \in \mathbb{Z}\right\}$ be the subgroup of Aut $G$ generated by $A_{1}$.

Let $M \geq 0$ be any nonnegative integer, and set $W=\left(A_{1}^{*}\right)^{M} H^{\perp}$. Note that $W \subsetneq\left(A_{1}^{*}\right) W$, because $A_{1}$ is expansive. We shall be particularly interested in the set $\left(A_{1}^{*} W\right) \backslash W$. Informally speaking, $W$ is like a disk centered at the origin, so that $\left(A_{1}^{*} W\right) \backslash W$ is like an annulus. Our algorithm, based on similar constructions for $\mathbb{R}^{d}$ in $[10,11]$, will translate subsets of $W$ into $\left(A_{1}^{*} W\right) \backslash W$ until what remains is a wavelet collection of sets.

Let $N \geq 1$ be a positive integer, which ultimately will be the number of wavelet generators. Let $\Omega_{1,0}, \ldots, \Omega_{N, 0} \subseteq W$ be measurable sets, each of which is $(\tau, \mathcal{D})$-congruent to $H^{\perp}$, and let $\widetilde{\Omega}_{0}=\bigcup_{j=1}^{N} \Omega_{j, 0}$. We do not require the sets $\Omega_{j, 0}$ to be disjoint, but we assume that there is some integer $\ell \geq 0$ such that $\left(A_{1}^{*}\right)^{-\ell} H^{\perp} \subseteq \widetilde{\Omega}_{0}$. Thus, the union of all the sets $\Omega_{j, 0}$ contains a neighborhood of the origin. that

For each $j \in\{1, \ldots, N\}$, let $T_{j}: W \rightarrow\left(A_{1}^{*} W\right) \backslash W$ be a measurable injective function such

$$
\forall \gamma \in W, \quad T_{j}(\gamma)-\gamma+\theta_{\mathcal{D}}(\gamma) \in \mathcal{D} \cap\left[\left(A_{1}^{*} W\right) \backslash W\right],
$$

where $\theta_{\mathcal{D}}(\gamma)$ is the unique element of $\mathcal{D} \cap\left(\gamma+H^{\perp}\right)$, as in Definition 2.1. Thus, because $\mathcal{D} \cap\left(A_{1}^{*} W\right)$ is finite, $T_{j}$ slices $W$ into finitely many measurable pieces $U_{j, n}$, each of which it translates by some element of the form $\sigma_{j, n}-\sigma_{j, n}^{\prime}$, so that each piece lands in the "annulus" 
$\left(A_{1}^{*} W\right) \backslash W$, and in such a way that the translated pieces do not overlap. Moreover, $\sigma_{j, n}^{\prime} \in \mathcal{D} \cap W$ and $\sigma_{j, n} \in \mathcal{D} \cap\left[\left(A_{1}^{*} W\right) \backslash W\right]$, with $U_{j, n} \subseteq \sigma_{j, n}^{\prime}+H^{\perp}$, so that $T_{j}\left(U_{j, n}\right) \subseteq \sigma_{j, n}+H^{\perp}$.

Finally, we require the following compatibility condition between the functions $T_{j}$ and the sets $\Omega_{j, 0}$. For any distinct $j, k \in\{1, \ldots, N\}$, assume that either

$$
T_{j} W \cap T_{k} W=\emptyset,
$$

or

$$
T_{j}=T_{k} \quad \text { and } \quad \Omega_{j, 0} \cap \Omega_{k, 0}=\emptyset .
$$

Given the initial data described above, the algorithm proceeds as follows. First, for each $j \in\{1, \ldots, N\}$, define

$$
\begin{array}{ll}
\Lambda_{j, 1}^{\prime}=\Omega_{j, 0} \cap\left[\bigcup_{n \geq 1}\left(A_{1}^{*}\right)^{-n} \widetilde{\Omega}_{0}\right], & \Lambda_{j, 1}^{\prime \prime}=\Omega_{j, 0} \cap\left[\bigcup_{k=1}^{j-1} \Omega_{k, 0}\right], \\
\Lambda_{j, 1}=\Lambda_{j, 1}^{\prime} \cup \Lambda_{j, 1}^{\prime \prime}, & \text { and } \quad \Omega_{j, 1}=\left(\Omega_{j, 0} \backslash \Lambda_{j, 1}\right) \cup T_{j} \Lambda_{j, 1} .
\end{array}
$$

In addition, define

$$
\widetilde{\Lambda}_{1}^{\prime}=\bigcup_{k=1}^{N} \Lambda_{k, 1}^{\prime}
$$

Next, for every $m \geq 1$ and $1 \leq j \leq N$, define

$$
\Lambda_{j, m+1}=\Omega_{j, m} \cap\left[\bigcup_{n \geq 1}\left(A_{1}^{*}\right)^{-n} \widetilde{\Omega}_{m}\right] \quad \text { and } \quad \Omega_{j, m+1}=\left(\Omega_{j, m} \backslash \Lambda_{j, m+1}\right) \cup T_{j} \Lambda_{j, m+1} \text {, }
$$

where

$$
\widetilde{\Omega}_{m}=\bigcup_{k=1}^{N} \Omega_{k, m}
$$

Finally, for every $j \in\{1, \ldots, N\}$, define

$$
\Lambda_{j}=\bigcup_{m \geq 1} \Lambda_{j, m}, \quad \Omega_{j}=\left(\Omega_{j, 0} \backslash \Lambda_{j}\right) \cup T_{j} \Lambda_{j}, \quad \text { and } \quad \widetilde{\Omega}=\bigcup_{j=1}^{N} \Omega_{j} .
$$

Theorem 4.2 below will show that the $\left\{\Omega_{1}, \ldots, \Omega_{N}\right\}$ so constructed is a wavelet collection of sets.

The inductive definitions above make sense provided that each $\Lambda_{j, m}$ lies in $W$, the domain of $T_{j}$. To see that this is indeed the case, note that, by a simple induction on $m$, we have

$$
\Lambda_{j, m} \subseteq \Omega_{j, 0} \quad \text { and } \quad \Omega_{j, m} \subseteq\left[\left(A_{1}^{*} W\right) \backslash W\right] \cup \Omega_{j, 0} .
$$

Thus, $\Lambda_{j, m} \subseteq \Omega_{j, 0} \subseteq W$, as desired. By another simple induction, we also observe that

$$
\Omega_{j, m}=\left(\Omega_{j, 0} \backslash \bigcup_{i=1}^{m} \Lambda_{j, i}\right) \cup T_{j}\left(\bigcup_{i=1}^{m} \Lambda_{j, i}\right)
$$


The algorithm is never vacuous, in the sense that given $G, H, \mathcal{D}$, and $A_{1}$, a choice of $M$, $N,\left\{T_{j}\right\}$, and $\left\{\Omega_{j, 0}\right\}$ satisfying our criteria always exists. For example, note that $H^{\perp} \subsetneq A_{1}^{*} H^{\perp}$ implies that $\left(A_{1}^{*} H^{\perp}\right) \backslash H^{\perp}$ must contain an element $\sigma_{1}$ of $\mathcal{D}$. Thus, letting $\sigma_{0}^{\prime}$ denote the unique element of $\mathcal{D} \cap H^{\perp}$, we may choose $M=0$ (so $W=H^{\perp}$ ), $N=1, T_{1}(\gamma)=\gamma-\sigma_{0}^{\prime}+\sigma_{1}$ for all $\gamma \in W$, and $\Omega_{1,0}=H^{\perp}$. Of course, many other choices are possible as well, as we shall see in Section 5 .

\subsection{Validity of the algorithm}

Intuitively, the algorithm just described should generate a wavelet collection of sets for the following reasons. The structure of $T_{j}$ will force each $\left\{\Omega_{j, m+1}\right\}$ to be $(\tau, \mathcal{D})$-congruent to $\Omega_{j, m}$ and hence, inductively, to $H^{\perp}$. At the same time, each $\widetilde{\Omega}_{m}$ shares with $\widetilde{\Omega}_{0}$ the property that $\bigcup_{n \in \mathbb{Z}}\left(A_{1}^{*}\right)^{n} \widetilde{\Omega}_{m}$ covers $\widehat{G}$ up to sets of measure zero. Meanwhile, the sets $\Lambda_{j, 1}^{\prime \prime}$ guarantee that the sets $\left\{\Omega_{j, 1}\right\}_{j=1}^{N}$ are pairwise disjoint; and conditions (4.2) and (4.3) extend that property to $\left\{\Omega_{j, m}\right\}_{j=1}^{N}$, for every $m \geq 1$. More generally, the sets $\Lambda_{j, m}$ are regions corresponding to overlaps between different dilates $\left(A_{1}^{*}\right)^{n} \Omega_{j, m}$. By translating them out to $\left(A_{1}^{*} W\right) \backslash W$, future overlaps should be smaller, and should vanish in the limit.

Obviously, the rigorous proof requires more detail, including the following lemma.

Lemma 4.1. Given $\left\{\Lambda_{j, 1}\right\},\left\{\Lambda_{j}\right\}$, and $\widetilde{\Omega}$ as defined in (4.4)-(4.7) above. Then for every $j=1, \ldots, N$,

$$
\Lambda_{j} \subseteq \Lambda_{j, 1} \cup\left[\bigcup_{n \geq 1}\left(A_{1}^{*}\right)^{-n} \widetilde{\Omega}\right] .
$$

Proof. By (4.7) and (4.9), we have $\Omega_{j, m} \subseteq \Omega_{j, 0} \cup T_{j} \Lambda_{j} \subseteq \Omega_{j, 0} \cup \Omega_{j}$, for any $m \geq 0$ and $1 \leq j \leq N$. It follows that $\widetilde{\Omega}_{m} \subseteq \widetilde{\Omega}_{0} \cup \widetilde{\Omega}$ for any $m \geq 1$. By (4.8) and the fact that $W \subseteq A_{1}^{*} W$, we have $\left(A_{1}^{*}\right)^{-n} \Omega_{j, m} \subseteq W$, and hence $\left(T_{j} \Lambda_{j}\right) \cap\left(A_{1}^{*}\right)^{-n} \widetilde{\Omega}_{m}=\emptyset$, for all $m \geq 0, n \geq 1$, and $1 \leq j \leq N$. Thus,

$$
\begin{aligned}
\Lambda_{j} & =\bigcup_{m \geq 1} \Lambda_{j, m}=\Lambda_{j, 1} \cup \bigcup_{m \geq 1}\left[\Omega_{j, m} \cap\left(\bigcup_{n \geq 1}\left(A_{1}^{*}\right)^{-n} \widetilde{\Omega}_{m}\right)\right] \\
& \subseteq \Lambda_{j, 1} \cup \bigcup_{m \geq 1}\left[\left(\Omega_{j, 0} \cup T_{j} \Lambda_{j}\right) \cap\left(\bigcup_{n \geq 1}\left(A_{1}^{*}\right)^{-n} \widetilde{\Omega}_{m}\right)\right] \\
& =\Lambda_{j, 1} \cup \bigcup_{m \geq 1}\left[\Omega_{j, 0} \cap\left(\bigcup_{n \geq 1}\left(A_{1}^{*}\right)^{-n} \widetilde{\Omega}_{m}\right)\right] \\
& \subseteq \Lambda_{j, 1} \cup\left[\Omega_{j, 0} \cap\left(\bigcup_{n \geq 1}\left(A_{1}^{*}\right)^{-n}\left(\widetilde{\Omega}_{0} \cup \widetilde{\Omega}\right)\right)\right] \subseteq \Lambda_{j, 1} \cup\left[\bigcup_{n \geq 1}\left(A_{1}^{*}\right)^{-n} \widetilde{\Omega}\right],
\end{aligned}
$$

where the final inclusion follows because $\Omega_{j, 0} \cap\left(\bigcup_{n \geq 1}\left(A_{1}^{*}\right)^{-n} \widetilde{\Omega}_{0}\right)=\Lambda_{j, 1}^{\prime} \subseteq \Lambda_{j, 1}$.

Theorem 4.2. Let $G$ be a $L C A G$ with compact open subgroup $H \subseteq G$, let $\mathcal{D} \subseteq \widehat{G}$ be a choice of coset representatives in $\widehat{G}$ for $\widehat{H}=\widehat{G} / H^{\perp}$, let $A_{1} \in$ Aut $G$ be an expansive automorphism, and set $\mathcal{A}=\left\{A_{1}^{n}: n \in \mathbb{Z}\right\}$. For a fixed nonnegative integer $M \geq 0$ define the set $W=\left(A_{1}^{*}\right)^{M} H^{\perp}$; 
and for a fixed positive integer $N \geq 1$ let $\left\{T_{j}: j=1, \ldots, N\right\}$ be a set of measurable injective functions

$$
T_{j}: W \rightarrow\left(A_{1}^{*} W\right) \backslash W, \quad j=1, \ldots, N
$$

satisfying (4.1). Consider a finite sequence $\Omega_{1,0}, \ldots, \Omega_{N, 0} \subseteq W$ of measurable subsets of $\widehat{G}$, each of which is $(\tau, \mathcal{D})$-congruent to $H^{\perp} \subseteq \widehat{G}$, and for which there is a nonnegative integer $\ell \geq 0$ such that

$$
\left(A_{1}^{*}\right)^{-\ell} H^{\perp} \subseteq \widetilde{\Omega}_{0}=\bigcup_{j=1}^{N} \Omega_{j, 0} .
$$

Assume that for every distinct pair $j, k \in\{1, \ldots, N\}$, either condition (4.2) or (4.3) holds. Then the sequence $\left\{\Omega_{1}, \ldots, \Omega_{N}\right\}$ produced by the algorithm (4.4)-(4.7) forms a wavelet collection of sets.

Proof. We shall use the criterion from Theorem 3.5 to prove this theorem.

(i) It follows easily from the definition of the maps $T_{j}$ that if $X$ is a measurable subset of $W$, then $T_{j}(X)$ is a measurable subset of $\widehat{G}$. The sets $\left\{\Omega_{j}\right\}$ are formed from the measurable sets $\left\{\Omega_{j, 0}\right\}$ using only complements, countable unions, countable intersections, and images under the maps $T_{j}$. Hence, each $\Omega_{j}$ is measurable.

(ii) We shall now show that for any $j=1, \ldots, n, \Omega_{j}$ is $(\tau, \mathcal{D})$-congruent to $H^{\perp} \subseteq \widehat{G}$.

Fix $j \in\{1, \ldots, N\}$. Let $I=\mathcal{D} \cap\left[\left(A_{1}^{*} W\right) \backslash W\right]$ and $I^{\prime}=\mathcal{D} \cap W$, both of which are finite sets. For each pair $\left(\sigma, \sigma^{\prime}\right) \in I \times I^{\prime}$, define

$$
U_{j, \sigma, \sigma^{\prime}}=\left\{\gamma \in \sigma^{\prime}+H^{\perp}: T_{j}(\gamma)=\gamma-\sigma^{\prime}+\sigma\right\} .
$$

Then by definition of $T_{j}$, each $\gamma \in W$ lies in exactly one $U_{j, \sigma, \sigma^{\prime}}$. Moreover, the translated sets $T_{j}\left(U_{j, \sigma, \sigma^{\prime}}\right)$ are pairwise disjoint, by the hypothesis that $T_{j}$ is injective.

For each $\left(\sigma, \sigma^{\prime}\right) \in I \times I^{\prime}$, define $V_{j, \sigma, \sigma^{\prime}}^{\prime}=\left(U_{j, \sigma, \sigma^{\prime}} \cap \Lambda_{j}\right)$ and $V_{j, \sigma, \sigma^{\prime}}=T_{j}\left(V_{j, \sigma, \sigma^{\prime}}^{\prime}\right)$. In addition, for every $\sigma^{\prime} \in I^{\prime}$, define $V_{j, \sigma^{\prime}}^{\prime}=V_{j, \sigma^{\prime}}=\left(\Omega_{j, 0} \backslash \Lambda_{j}\right) \cap\left(\sigma^{\prime}+H^{\perp}\right)$. Using the finite set $I^{\prime} \cup\left(I \times I^{\prime}\right)$ as an indexing set, we are now prepared to exhibit the $(\tau, \mathcal{D})$-congruence.

Because $\Lambda_{j} \subseteq \Omega_{j, 0},\left\{V_{j, \sigma, \sigma^{\prime}}^{\prime}:\left(\sigma, \sigma^{\prime}\right) \in I \times I^{\prime}\right\} \cup\left\{V_{j, \sigma^{\prime}}^{\prime}: \sigma^{\prime} \in I^{\prime}\right\}$ tiles $\Omega_{j, 0}$. By definition of $\Omega_{j},\left\{V_{j, \sigma, \sigma^{\prime}}:\left(\sigma, \sigma^{\prime}\right) \in I \times I^{\prime}\right\} \cup\left\{V_{j, \sigma^{\prime}}: \sigma^{\prime} \in I^{\prime}\right\}$ tiles $\Omega_{j}$. Moreover, $V_{j, \sigma, \sigma^{\prime}}-\sigma=V_{j, \sigma, \sigma^{\prime}}^{\prime}-\sigma^{\prime}$ and $V_{j, \sigma^{\prime}}=V_{j, \sigma^{\prime}}^{\prime}$. Therefore, by definition of $T_{j}, \Omega_{j}$ is $(\tau, \mathcal{D})$-congruent to $\Omega_{j, 0}$. Since $\Omega_{j, 0}$ is $(\tau, \mathcal{D})$-congruent to $H^{\perp}$, it follows that $\Omega_{j}$ is $(\tau, \mathcal{D})$-congruent to $H^{\perp}$.

(iii) Next, we shall show that for any $j, k \in\{1, \ldots, N\}$ and for any distinct $A, B \in \mathcal{A}$, the sets $A^{*} \Omega_{j}$ and $B^{*} \Omega_{k}$ are disjoint. Because $\mathcal{A}=\left\{A_{1}^{n}: n \in \mathbb{Z}\right\}$, it suffices to show that

$$
\Omega_{k} \cap\left(A_{1}^{*}\right)^{n} \Omega_{j}=\emptyset \quad \text { for all } j, k \in\{1, \ldots, N\} \text { and } n \geq 1 .
$$

Fix $j, k \in\{1, \ldots, N\}$ and $n \geq 1$, and suppose that there is some $\gamma \in \Omega_{k} \cap\left(A_{1}^{*}\right)^{n} \Omega_{j}$. Thus, $\gamma \in \Omega_{k}$ and $\left(A_{1}^{*}\right)^{-n} \gamma \in \Omega_{j}$.

If $\gamma \in \Omega_{k, 0}$, then because $\Omega_{k, 0} \subseteq W \subseteq\left(A_{1}^{*}\right)^{n} W$, we have $\left(A_{1}^{*}\right)^{-n} \gamma \in W \cap \Omega_{j} \subseteq \Omega_{j, 0}$, by (4.7) and the fact that the image of $T_{j}$ is disjoint from $W$. Thus,

$$
\left(A_{1}^{*}\right)^{-n} \gamma \in \Omega_{j, 0} \cap\left(A_{1}^{*}\right)^{-n} \Omega_{k, 0} \subseteq \Omega_{j, 0} \cap\left(A_{1}^{*}\right)^{-n} \widetilde{\Omega}_{0} \subseteq \Lambda_{j, 1} \subseteq \Lambda_{j} .
$$

Then $\left(A_{1}^{*}\right)^{-n} \gamma \notin \Omega_{j}$, which is a contradiction. 
On the other hand, if $\gamma \notin \Omega_{k, 0}$, then $\gamma \in T_{k} \Lambda_{k}$ by (4.7), so that $\gamma \in T_{k} \Lambda_{k, m}$ for some $m \geq 1$. Note that $\gamma \in A_{1}^{*} W$, so that $\left(A_{1}^{*}\right)^{-n} \gamma \in W$, and therefore

$$
\left(A_{1}^{*}\right)^{-n} \gamma \in W \cap \Omega_{j} \subseteq \Omega_{j, 0} \backslash \Lambda_{j} \subseteq \Omega_{j, 0} \backslash\left(\bigcup_{i=1}^{m} \Lambda_{j, i}\right) \subseteq \Omega_{j, m},
$$

by (4.7) and (4.9). Also note that $\gamma \in T_{k} \Lambda_{k, m} \subseteq \Omega_{k, m} \subseteq \widetilde{\Omega}_{m}$. Thus, we have $\gamma \in \widetilde{\Omega}_{m}$ and $\left(A_{1}^{*}\right)^{-n} \gamma \in \Omega_{j, m}$; hence,

$$
\left(A_{1}^{*}\right)^{-n} \gamma \in \Omega_{j, m} \cap\left(A_{1}^{*}\right)^{-n} \widetilde{\Omega}_{m} \subseteq \Lambda_{j, m+1} \subseteq \Lambda_{j},
$$

contradicting the assumption that $\left(A_{1}^{*}\right)^{-n} \gamma \in \Omega_{j}$.

(iv) Now we shall show that for any distinct pairs $(A, j),(B, k) \in \mathcal{A} \times\{1, \ldots, N\}$, the sets $A^{*} \Omega_{j}$ and $B^{*} \Omega_{k}$ are disjoint. In light of (iii), we may assume that $A=B$, and therefore it suffices to show that for any distinct $j, k \in\{1, \ldots, N\}$, the sets $\Omega_{j}$ and $\Omega_{k}$ are disjoint.

Without loss, assume that $k<j$. If $T_{j}=T_{k}$, then by hypothesis (4.3), $\Omega_{j, 0} \cap \Omega_{k, 0}=\emptyset$, and therefore $\Lambda_{j} \cap \Lambda_{k}=\emptyset$, by (4.8). Because $T_{j}=T_{k}$ is injective, it follows that $T_{j} \Lambda_{j} \cap T_{k} \Lambda_{k}=\emptyset$. Moreover, the image of $T_{j}$ does not intersect $\Omega_{j, 0}, \Omega_{k, 0} \subseteq W$. It follows that $\Omega_{j} \cap \Omega_{k}=\emptyset$.

On the other hand, if $T_{j} \neq T_{k}$, then by hypothesis (4.2), $T_{j} W \cap T_{k} W=\emptyset$, so that $T_{j} \Lambda_{j} \cap$ $T_{k} \Lambda_{k}=\emptyset$. Moreover, $\Omega_{j, 0} \cap \Omega_{k, 0} \subseteq \Lambda_{j, 1}^{\prime \prime} \subseteq \Lambda_{j}$ by (4.4) and (4.5), so that

$$
\left(\Omega_{j, 0} \backslash \Lambda_{j}\right) \cap\left(\Omega_{k, 0} \backslash \Lambda_{k}\right)=\emptyset .
$$

Again by the fact that the images of $T_{j}$ and $T_{k}$ do not intersect $\Omega_{j, 0}, \Omega_{k, 0} \subseteq W$, it follows that $\Omega_{j} \cap \Omega_{k}=\emptyset$.

(v) Finally, we need to show that $\left\{A^{*} \Omega_{j}: A \in \mathcal{A}, 1 \leq j \leq N\right\}$ covers $\widehat{G}$ up to sets of measure zero. Let $Y=\bigcap_{n \in \mathbb{Z}}\left(A_{1}^{*}\right)^{n} H^{\perp}$. Because $v\left(\left(A_{1}^{*}\right)^{n} H^{\perp}\right)=\left|A_{1}\right|^{n}$, we have

$$
v(Y) \leq \inf \left\{\left|A_{1}\right|^{n}: n \in \mathbb{Z}\right\}=0 .
$$

Therefore, it will suffice to show that

$$
\widehat{G} \backslash Y \subseteq \bigcup_{A \in \mathcal{A}} A^{*} \widetilde{\Omega}
$$

Given $\gamma \in \widehat{G} \backslash Y$, let $J_{\gamma}=\left\{n \in \mathbb{Z}:\left(A_{1}^{*}\right)^{-n} \gamma \in \widetilde{\Omega}_{0}\right\}$. We shall need a minimal element of $J_{\gamma}$, and so we must show that $J_{\gamma}$ is nonempty and bounded below.

By the hypothesis that $\left(A_{1}^{*}\right)^{-\ell} H^{\perp} \subseteq \widetilde{\Omega}_{0}$ and because $A_{1}$ is expansive,

$$
\widehat{G}=\bigcup_{n \geq 0}\left(A_{1}^{*}\right)^{n-\ell} H^{\perp} \subseteq \bigcup_{n \geq 0}\left(A_{1}^{*}\right)^{n} \widetilde{\Omega}_{0},
$$

and therefore $J_{\gamma}$ is nonempty. On the other hand, suppose that for every integer $n \in \mathbb{Z}$, there is some integer $i_{n} \leq n$ such that $i_{n} \in J_{\gamma}$, that is, $\left(A_{1}^{*}\right)^{-i_{n}} \gamma \in \widetilde{\Omega}_{0}$. Then because $\widetilde{\Omega}_{0} \subseteq\left(A_{1}^{*}\right)^{M} H^{\perp}$ and because $A_{1}$ is expansive, we have

$$
\gamma \in\left(A_{1}^{*}\right)^{i_{n}} \widetilde{\Omega}_{0} \subseteq\left(A_{1}^{*}\right)^{i_{n}+M} H^{\perp} \subseteq\left(A_{1}^{*}\right)^{n+M} H^{\perp} .
$$

Thus, $\gamma \in \bigcap_{n \in \mathbb{Z}}\left(A_{1}^{*}\right)^{n+M} H^{\perp}=Y$, which is a contradiction. It follows that $J_{\gamma}$ is bounded below. Hence, it makes sense to define $i=\min J_{\gamma}$. 
We claim that $\left(A_{1}^{*}\right)^{-i} \gamma \in \widetilde{\Omega}_{0} \backslash \widetilde{\Lambda}_{1}^{\prime}$. Indeed, we have $\left(A_{1}^{*}\right)^{-i} \gamma \in \widetilde{\Omega}_{0}$ because $i \in J_{\gamma}$. On the other hand, if $\left(A_{1}^{*}\right)^{-i} \gamma \in \widetilde{\Lambda}_{1}^{\prime}$, then by definition of $\widetilde{\Lambda}_{1}^{\prime}$, we have $\left(A_{1}^{*}\right)^{-i} \gamma \in\left(A_{1}^{*}\right)^{-n} \widetilde{\Omega}_{0}$ for some $n \geq 1$. Therefore, $\left(A_{1}^{*}\right)^{-(i-n)} \gamma \in \widetilde{\Omega}_{0}$, so that $i-n \in J_{\gamma}$, which contradicts the minimality of $i$ and proves our claim.

Let $j \in\{1, \ldots, N\}$ be the smallest index such that $\left(A_{1}^{*}\right)^{-i} \gamma \in \Omega_{j, 0}$; note that $j$ exists because $\left(A_{1}^{*}\right)^{-i} \gamma \in \widetilde{\Omega}_{0}$.

If $\left(A_{1}^{*}\right)^{-i} \gamma \in \Omega_{j}$, then $\gamma \in\left(A_{1}^{*}\right)^{i} \Omega_{j}$, and we are done.

Otherwise, $\left(A_{1}^{*}\right)^{-i} \gamma \in \Lambda_{j}$. However, $\left(A_{1}^{*}\right)^{-i} \gamma \notin \Lambda_{j, 1}^{\prime}$, by the claim above; and $\left(A_{1}^{*}\right)^{-i} \gamma \notin$ $\Lambda_{j, 1}^{\prime \prime}$ by our choice of $j$. Thus, $\left(A_{1}^{*}\right)^{-i} \gamma \in \Lambda_{j} \backslash \Lambda_{j, 1}$. Therefore, by Lemma 4.1, there is some $n \geq 1$ for which $\left(A_{1}^{*}\right)^{-i} \gamma \in\left(A_{1}^{*}\right)^{-n} \widetilde{\Omega}$. Hence, $\gamma \in\left(A_{1}^{*}\right)^{i-n} \Omega$, and the theorem is proved.

\section{Examples of sets of wavelet generators}

Theorems 3.5 and 4.2 together provide an algorithm for generating many wavelet orthonormal bases for $L^{2}(G)$ for a group $G$ with the properties specified in Theorem 4.2. In this section, we shall examine several examples of such wavelets. Other examples can be found in [14].

\subsection{Haar/Shannon wavelets on $G$}

Let $G$ be a LCAG with compact open subgroup $H$, let $\mathcal{D}$ be a choice set of coset representatives in $\widehat{G}$ for $\widehat{G} / H^{\perp}$, and let $A_{1}$ be an expansive automorphism of $G$.

Take $M=0$, so that $W=H^{\perp}$, set $N=\left|A_{1}\right|-1 \geq 1$, and let $\sigma_{1}, \ldots, \sigma_{N}$ be the $N$ elements of $\mathcal{D} \cap\left[\left(A_{1}^{*} H^{\perp}\right) \backslash H^{\perp}\right]$. For each $j=1, \ldots, N$, define $T_{j}(\gamma)=\gamma-\sigma_{0}^{\prime}+\sigma_{j}$, where $\sigma_{0}^{\prime}$ denotes the unique element of $\mathcal{D} \cap H^{\perp}$, and define $\Omega_{j, 0}=H^{\perp}$. Note that $\left\{H^{\perp}, T_{1} H^{\perp}, \ldots, T_{N} H^{\perp}\right\}$ is a set of $\left|A_{1}\right|=N+1$ compact open sets which together tile $A_{1}^{*} H^{\perp}$. See Figure 2 for a diagram of $\left\{T_{j}\right\}$ and $\Omega_{j, 0}(j=1,2,3)$ in the case that $\left|A_{1}\right|=4$.

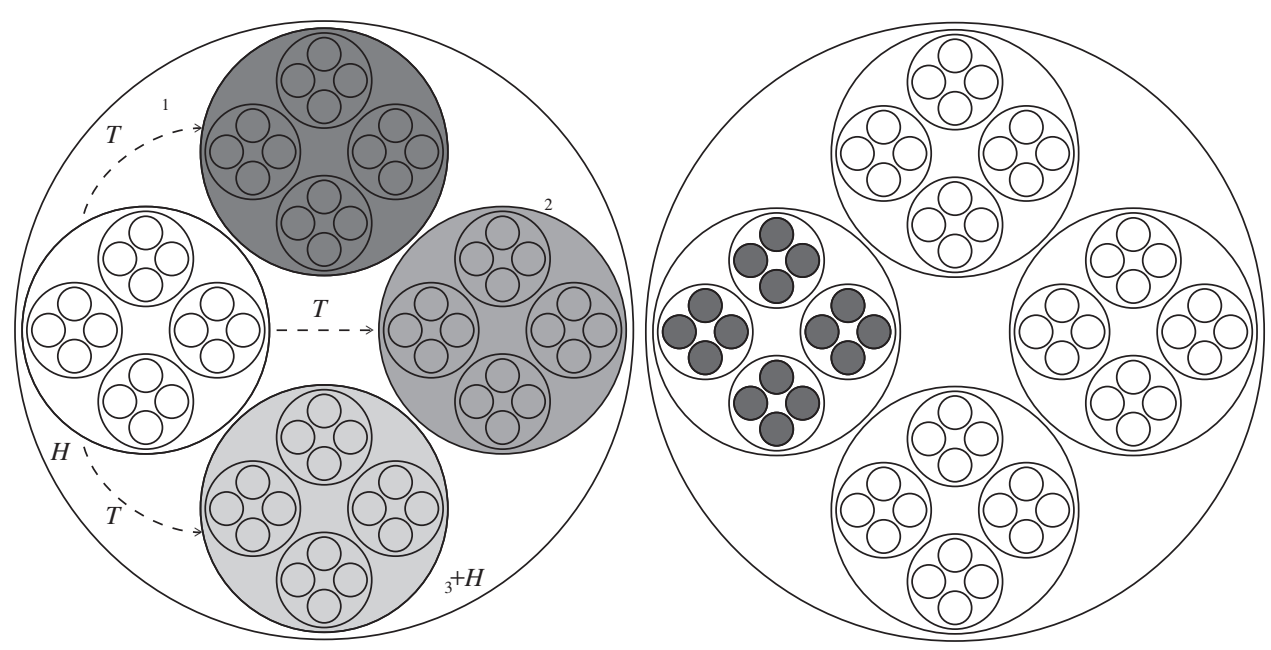

FIGURE 2 The maps $T_{j}$ and the sets $\Omega_{1,0}=\Omega_{2,0}=\Omega_{3,0}$ of Example 5.1, for $\left|A_{1}\right|=4$. 
When we apply the algorithm from Section 4 to this data, we obtain, up to sets of measure zero,

$$
\Omega_{j}=\sigma_{j}-\sigma_{0}^{\prime}+H^{\perp}=\sigma_{j}+H^{\perp} \quad \text { for all } j \in\{1, \ldots, N\} .
$$

To see this, first note that for $j \geq 2$, we have $\Lambda_{j, 1}^{\prime \prime}=H^{\perp}$, so that $\Lambda_{j}=H^{\perp}$, and hence $\Omega_{j}=T_{j} H^{\perp}$, as claimed (see Figure 3).

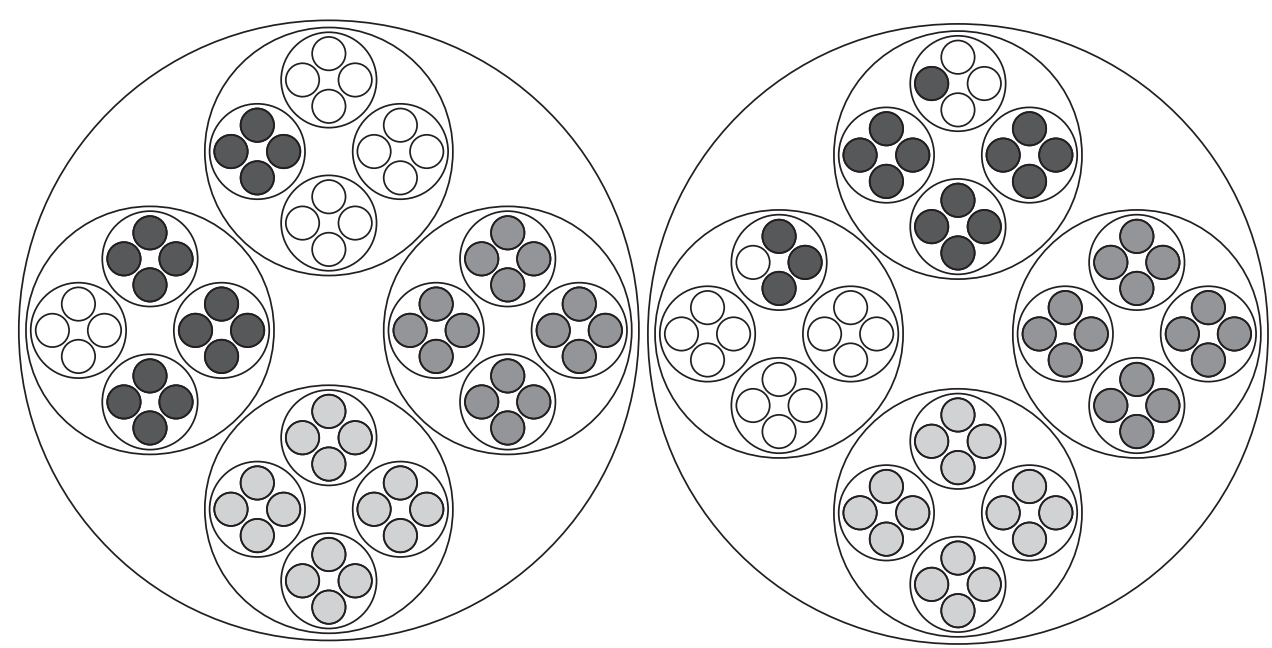

FIGURE 3 The sets $\Omega_{j, m}(j=1,2,3, m=1,2)$ of Example 5.1, for $\left|A_{1}\right|=4$.

Meanwhile, $\Lambda_{1,1}=\left(A_{1}^{*}\right)^{-1} H^{\perp}$, and the observations about $\Lambda_{j}$ for $j \geq 2$ imply that

$$
\bigcup_{j=2}^{N}\left(A_{1}^{*}\right)^{-1} T_{j} H^{\perp} \subseteq \Lambda_{1,2} .
$$

Because $\left\{H^{\perp}, T_{1} H^{\perp}, \ldots, T_{N} H^{\perp}\right\}$ tiles $A_{1}^{*} H^{\perp}$, it follows that

$$
H^{\perp} \cap \Omega_{1,2} \subseteq \gamma_{2}+\left(A_{1}^{*}\right)^{-1} H^{\perp}, \quad \text { where } \gamma_{2}=\left(A_{1}^{*}\right)^{-1} T_{1}(0) .
$$

By induction on $m$, we obtain

$$
H^{\perp} \cap \Omega_{1, m} \subseteq \gamma_{m}+\left(A_{1}^{*}\right)^{-(m-1)} H^{\perp}, \quad \text { where } \gamma_{m}=\left(A_{1}^{*}\right)^{-1} T_{1}\left(\gamma_{m-1}\right) .
$$

See Figure 3 for a diagram of $\Omega_{j, m}(j=1,2,3, m=1,2)$ in the case that $\left|A_{1}\right|=4$.

Thus, $v\left(\Lambda_{1}\right)=1-\inf \left\{\left|A_{1}\right|^{-(m-1)}: m \geq 2\right\}=1$, so that $\Lambda_{1}=H^{\perp}$, up to sets of measure zero. The characterization of $\left\{\Omega_{j}: j=1, \ldots, N\right\}$ in (5.1) then follows. Note that by that characterization, the set $\mathcal{D}$ is in this case irrelevant to the ultimate set of wavelet generators. The wavelets $\left\{\psi_{j}: j=1, \ldots, N\right\}$ themselves are described by the following proposition.

Proposition 5.1. Let $G$ be a $L C A G$ with compact open subgroup $H \subseteq G$, let $\mathcal{D} \subseteq \widehat{G}$ be a choice of coset representatives in $\widehat{G}$ for $\widehat{G} / H^{\perp}$, let $A_{1} \in$ Aut $G$ be an expansive automorphism, and set $N=\left|A_{1}\right|-1 \geq 1$. Denote by $\left\{\sigma_{j}: j=1, \ldots, N\right\}$ the $N$ elements of $\mathcal{D} \cap\left[\left(A_{1}^{*} H^{\perp}\right) \backslash\right.$ $H^{\perp}$ ], write $\Omega_{j}=\sigma_{j}+H^{\perp}$ for $j=1, \ldots, N$, and define $\psi_{j} \in L^{2}(G)$ by $\widehat{\psi}_{j}=\mathbf{1}_{\Omega_{j}}$. Then $\left\{\psi_{1}, \ldots, \psi_{N}\right\}$ is a set of wavelet generators, and, for all $j=1, \ldots, N$ and $x \in G$,

$$
\psi_{j}(x)=\left(x, \sigma_{j}\right) \mathbf{1}_{H}(x) .
$$


Moreover, $\psi_{j}$ is constant on every open set of the form $c+A_{1}^{-1} H$.

Proof. By the preceding argument, $\left\{\Omega_{1}, \ldots, \Omega_{N}\right\}$ is the output of algorithm (4.4)-(4.7). By Theorem 4.2, $\left\{\psi_{1}, \ldots, \psi_{N}\right\}$ is a set of wavelet generators. We compute

$$
\psi_{j}(x)=\int_{\widehat{G}} \mathbf{1}_{\sigma_{j}+H^{\perp}}(\gamma)(x, \gamma) d \gamma=\int_{\sigma_{j}+H^{\perp}}(x, \gamma) d \gamma=\left(x, \sigma_{j}\right) \int_{H^{\perp}}(x, \beta) d \beta,
$$

by the change of variables $\gamma=\sigma_{j}+\beta$. If $x \in H$, then $(x, \beta)=1$ for all $\beta \in H^{\perp}$, so that the right side of (5.2) is $\left(x, \sigma_{j}\right)$, as desired. On the other hand, if $x \notin H$, then the integral is zero; see the discussion following (3.2).

Finally, let $c \in G$, and pick any $x, y \in c+A_{1}^{-1} H$. Then $y-x \in A_{1}^{-1} H$; moreover, $x$ lies in $H$ if and only if $y$ does also. Thus,

$$
\psi_{j}(y)=\left(y, \sigma_{j}\right) \mathbf{1}_{H}(y)=\left(y-x, \sigma_{j}\right)\left(x, \sigma_{j}\right) \mathbf{1}_{H}(x)=\left(y-x, \sigma_{j}\right) \psi_{j}(x) .
$$

However, $\left(y-x, \sigma_{j}\right)=\left(A_{1}(y-x),\left(A_{1}^{*}\right)^{-1} \sigma_{j}\right)$, and by hypothesis, $A_{1}(y-x) \in H$ and $\left(A_{1}^{*}\right)^{-1} \sigma_{j} \in H^{\perp}$. Thus, $\psi_{j}(y)=\psi_{j}(x)$, and $\psi_{j}$ is constant on $c+A_{1}^{-1} H$, as claimed.

In [14], it was observed that the wavelets $\left\{\psi_{1}, \ldots, \psi_{N}\right\}$ defined by $\psi_{j}=\check{\mathbf{1}}_{\Omega_{j}}$ are Shannon wavelets in the sense that each $\Omega_{j}$ is a simple translation of the original "fundamental domain" $H^{\perp}$. On the other hand, by Proposition 5.1, each $\psi_{j}$ is a step function with compact support, analogous to the usual Haar wavelet. (The functions $\psi_{j}$ are also continuous, which is possible because $G$ is a totally disconnected topological space.) Thus, although the Haar and Shannon wavelets lie at opposite extremes in the family of wavelets over $\mathbb{R}^{d}$, their analogues over $G$ are one and the same.

As a special case, let $p$ be a prime number, and consider $G=\mathbb{Q}_{p}$, as in Example 2.10. Let $A_{1}$ be the multiplication-by-1/p automorphism, which is expansive. Let $\mathcal{D}$ be any set of coset representatives in $\widehat{G}$ for $\widehat{G} / H^{\perp}=\mathbb{Q}_{p} / \mathbb{Z}_{p}$. For example, we could use Kozyrev's set $\mathcal{D}_{\mathrm{Koz}}=\left\{\frac{m}{p^{e}}: e \geq 1,0 \leq m \leq p^{e}-1\right\}$, which is the set generated by Lemma 2.7 using $\rho_{0}=0, \rho_{1}=1, \ldots, \rho_{p-1}=p-1$; but, as observed above, the Shannon wavelets produced in the end will be the same regardless of $\mathcal{D}$.

By (5.1), the wavelet sets generated by the algorithm are

$$
\Omega_{j}=j / p+\mathbb{Z}_{p} \quad \text { for } j \in\{1, \ldots, p-1\} .
$$

As observed in [14], one can compute that the wavelets $\psi_{j}=\check{\mathbf{1}}_{\Omega_{j}}$ are precisely the Haar wavelets constructed by Kozyrev [36, Theorem 2]. (On the other hand, our translation operators are different from his, so that the orthonormal basis is different, if only in that some of the elements of ours are scalar multiples of his.)

Similarly, if we let $G=\mathbb{F}_{2}((t))$, as in Example 2.11, then the data above gives the (single) wavelet set $\Omega_{1}=1 / t+\mathbb{F}_{2}[[t]]$, and the corresponding wavelet is precisely the Haar wavelet computed by Lang [39].

However, even for the Haar/Shannon wavelets, our construction is broader than those of Kozyrev and Lang, because it applies to any group $G$ satisfying our hypotheses (such as finite products or finite extensions of $\mathbb{Q}_{p}$ or of $\left.\mathbb{F}_{p}((t))\right)$, and because it works for any given expansive automorphism.

For example, let $G=\mathbb{Q}_{2} \times \mathbb{Q}_{3}$, and let $A_{1}$ be multiplication-by-(1/4,1/3). Then $A_{1}$ is an expansive automorphism with respect to the compact open subgroup $H=\mathbb{Z}_{2} \times \mathbb{Z}_{3}$, and 
$\left|A_{1}\right|=12$. The Haar/Shannon wavelet sets in $\widehat{G}=\mathbb{Q}_{2} \times \mathbb{Q}_{3}$ are the eleven sets of the form

$$
\left(j / 4+\mathbb{Z}_{2}\right) \times\left(k / 3+\mathbb{Z}_{3}\right)
$$

for $0 \leq j \leq 3$ and $0 \leq k \leq 2$, with $j$ and $k$ not both zero.

Alternatively, let $G=\mathbb{Q}_{2}(\sqrt{2})$, the field extension of $\mathbb{Q}_{2}$ obtained by adjoining $\sqrt{2}$, and let $H=\mathbb{Z}_{2}[\sqrt{2}]$ be the ring of integers in this field. Let $A_{1}$ be multiplication-by-2, which is an expansive automorphism, this time with $\left|A_{1}\right|=4$. We have $\widehat{G}=G$, with duality given by $(x, \gamma)=\exp (2 \pi i \operatorname{Tr}(x \gamma))$, where $\operatorname{Tr}: \mathbb{Q}_{2}(\sqrt{2}) \rightarrow \mathbb{Q}_{2}$ is the trace map $\operatorname{Tr}(y+z \sqrt{2})=2 y$ for $y, z \in \mathbb{Q}_{2}$. Note that $H^{\perp}=\left(2^{-3 / 2}\right) \mathbb{Z}_{2}[\sqrt{2}]$ under this duality. The Haar/Shannon wavelet sets in $\widehat{G}$ are the three sets

$2^{-5 / 2}+\left(2^{-3 / 2}\right) \mathbb{Z}_{2}[\sqrt{2}], \quad 2^{-5 / 2}+2^{-2}+\left(2^{-3 / 2}\right) \mathbb{Z}_{2}[\sqrt{2}], \quad$ and $\quad 2^{-2}+\left(2^{-3 / 2}\right) \mathbb{Z}_{2}[\sqrt{2}]$.

\subsection{Single wavelets on $G$}

Let $G$ be a LCAG with compact open subgroup $H$, let $\mathcal{D}$ be a choice of coset representatives in $\widehat{G}$ for $\widehat{G} / H^{\perp}$, and let $A_{1}$ be an expansive automorphism of $G$.

Take $M=0$, so that $W=H^{\perp}$, set $N=1$, and let $\sigma_{1}$ be any one of the $\left|A_{1}\right|-1$ elements of $\mathcal{D} \cap\left[\left(A_{1}^{*} H^{\perp}\right) \backslash H^{\perp}\right]$. Define $T_{1}(\gamma)=\gamma-\sigma_{0}^{\prime}+\sigma_{1}$, where $\sigma_{0}^{\prime}$ denotes the unique element of $\mathcal{D} \cap H^{\perp}$, and define $\Omega_{1,0}=H^{\perp}$. See Figure 4 for a diagram of $T_{1}$ and $\Omega_{1,0}$ in the case that $\left|A_{1}\right|=4$.

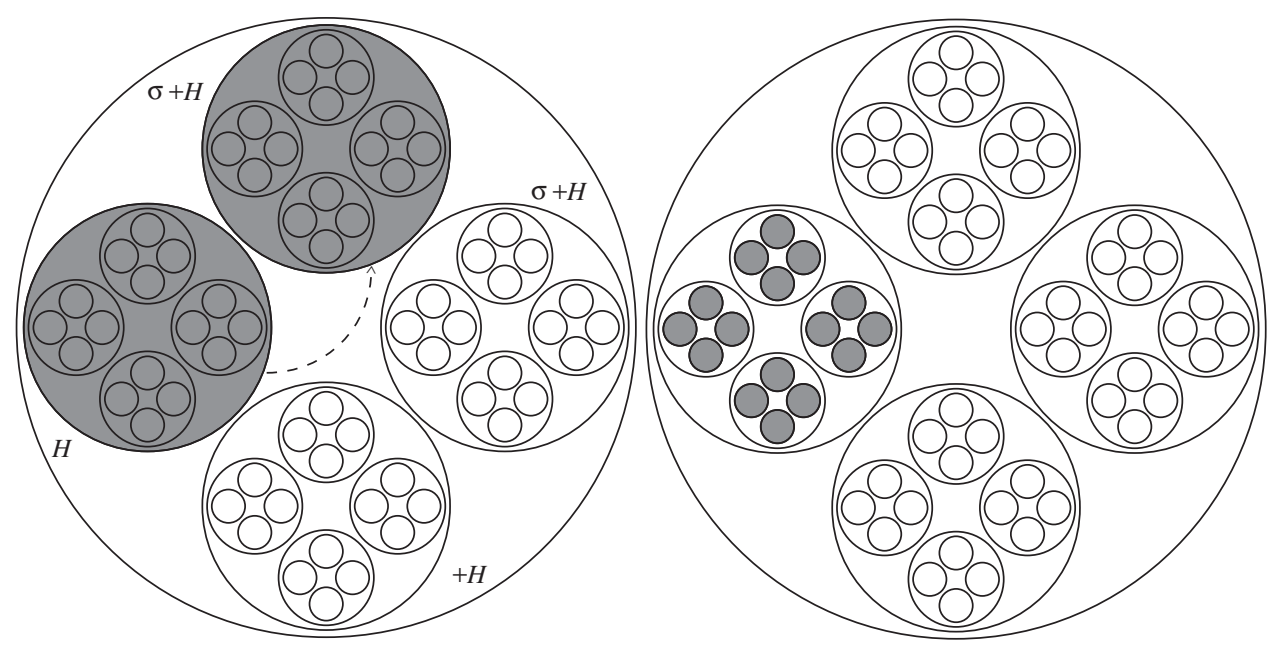

FIGURE 4 The map $T_{1}$ and set $\Omega_{1,0}$ of Example 5.2, for $\left|A_{1}\right|=4$.

A simple induction shows that for every $m \geq 1$,

$$
\Lambda_{1, m}=\left[\sum_{i=1}^{m-1}\left(A_{1}^{*}\right)^{-i}\left(\sigma_{1}-\sigma_{0}^{\prime}\right)\right]+\left(A_{1}^{*}\right)^{-m} H^{\perp} .
$$

If we think of $H^{\perp}$ as a "disk" of measure 1 , then each $\Lambda_{1, m}$ is a "disk" of measure $\left|A_{1}\right|^{-m}$. 
Moreover, these disks $\Lambda_{1, m}, m \geq 1$, are pairwise disjoint, and they approach the point

$$
c=\sum_{i=1}^{\infty}\left(A_{1}^{*}\right)^{-i}\left(\sigma_{1}-\sigma_{0}^{\prime}\right) \in H^{\perp} .
$$

See Figure 5 for some of the resulting sets $\Omega_{1, m}$.
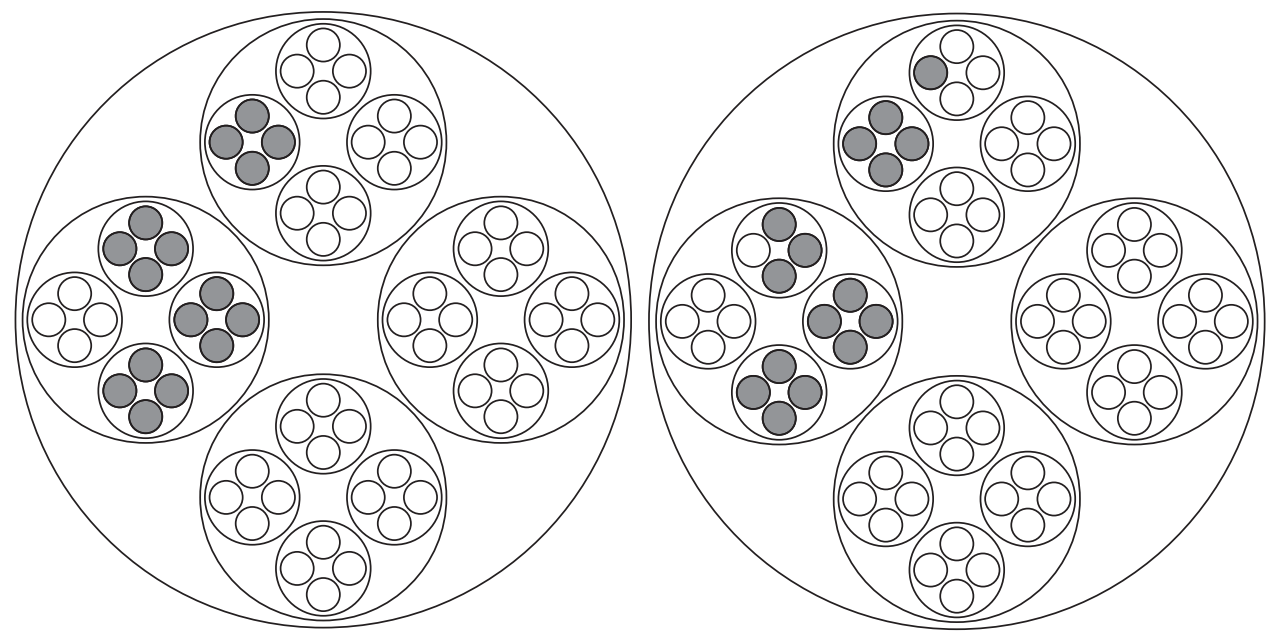

FIGURE $5 \Omega_{1,1}$ and $\Omega_{1,2}$ of Example 5.2.

Thus, $\Lambda_{1}$ is a countable union of such disks, and $v\left(\Lambda_{1}\right)=\left(\left|A_{1}\right|-1\right)^{-1}$. The wavelet set is then

$$
\Omega_{1}=\left(H^{\perp} \backslash \Lambda_{1}\right) \cup\left(\sigma_{1}-\sigma_{0}^{\prime}+\Lambda_{1}\right)
$$

For example, if $G=\mathbb{Q}_{5}$ and $A_{1}$ is multiplication-by-1/5, and if $\mathcal{D}$ contains $\sigma_{0}^{\prime}=0$ and $\sigma_{1}=1 / 5$, then we have

$$
\Lambda_{1, m}=\left(1+5+5^{2}+\ldots+5^{m-2}\right)+5^{m} \mathbb{Z}_{5}=-\frac{1}{4}-5^{m-1}+5^{m} \mathbb{Z}_{5}
$$

and therefore, since $-1 / 4+1 / 5=-1 / 20$, we have the wavelet set

$$
\Omega_{1}=\left[\mathbb{Z}_{5} \backslash \bigcup_{m=1}^{\infty}\left(-\frac{1}{4}-5^{m-1}+5^{m} \mathbb{Z}_{5}\right)\right] \cup \bigcup_{m=1}^{\infty}\left(-\frac{1}{20}-5^{m-1}+5^{m} \mathbb{Z}_{5}\right) .
$$

\subsection{Another single wavelet on $\mathbb{Q}_{3}$}

Let $G=\mathbb{Q}_{3}$, with compact open subgroup $H=\mathbb{Z}_{3}$, and let $A_{1}$ be multiplication-by-1/3, so that $A_{1}$ is expansive, with $\left|A_{1}\right|=3$. Identify $\widehat{G}$ as $\mathbb{Q}_{3}$ and $H^{\perp}$ as $\mathbb{Z}_{3}$, as in Example 2.10. Let $\mathcal{D}$ be a set of coset representatives in $\widehat{G}$ for $\widehat{G} / H^{\perp}$ including $\sigma_{0}^{\prime}=0, \sigma_{1}=1 / 3$, and $\sigma_{2}=2 / 3$.

Take $M=0$, so that $W=H^{\perp}$, set $N=1$, and let $\Omega_{1,0}=H^{\perp}$. For $\gamma \in H^{\perp}$, define

$$
T_{1}(\gamma)= \begin{cases}\gamma+2 / 3 & \text { if } \gamma \in 1+3 \mathbb{Z}_{3} \\ \gamma+1 / 3 & \text { if } \gamma \in\left(3 \mathbb{Z}_{3}\right) \cup\left(2+3 \mathbb{Z}_{3}\right)\end{cases}
$$




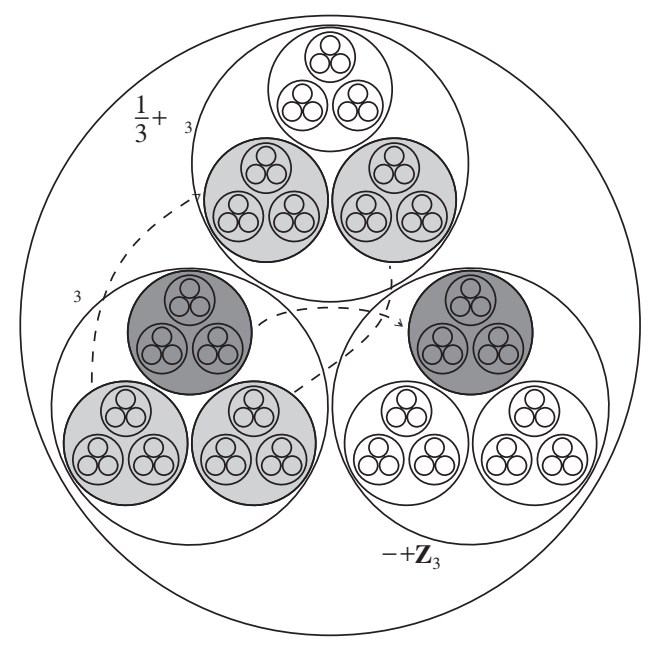

FIGURE 6 The map $T_{1}$ of Example 5.3.

as in Figure 6.

Clearly, $\Lambda_{1,1}=3 \mathbb{Z}_{3}$, which is a disk of measure $1 / 3$. By induction, it is easy to show that for $m \geq 1$,

$$
\Lambda_{1, m}= \begin{cases}2+1 \cdot 3+2 \cdot 3^{2}+1 \cdot 3^{3}+\cdots+1 \cdot 3^{m-2}+3^{m} \mathbb{Z}_{3} & \text { if } m \text { is odd } \\ 1+2 \cdot 3+1 \cdot 3^{2}+2 \cdot 3^{3}+\cdots+1 \cdot 3^{m-2}+3^{m} \mathbb{Z}_{3} & \text { if } m \text { is even }\end{cases}
$$

Note that the sums stop at $3^{m-2}$, so that $\Lambda_{1,1}=3 \mathbb{Z}_{3}$ and $\Lambda_{1,2}=1+3^{2} \mathbb{Z}_{3}$. Equivalently,

$$
\Lambda_{1, m}= \begin{cases}-5 / 8+3^{m-1}+3^{m} \mathbb{Z}_{3} & \text { if } m \text { is odd } \\ -7 / 8+3^{m-1}+3^{m} \mathbb{Z}_{3}, & \text { if } m \text { is even } .\end{cases}
$$

As in the previous example, $\Lambda_{1, m}$ is a disk of measure $1 / 3^{m}$, but it lies in $3 \mathbb{Z}_{3}$ if $m=1$, in $1+3 \mathbb{Z}_{3}$ if $m$ is even, and in $2+3 \mathbb{Z}_{3}$ if $m \geq 3$ is odd. See Figures $7-8$ for some of the resulting sets $\Omega_{1, m}$.

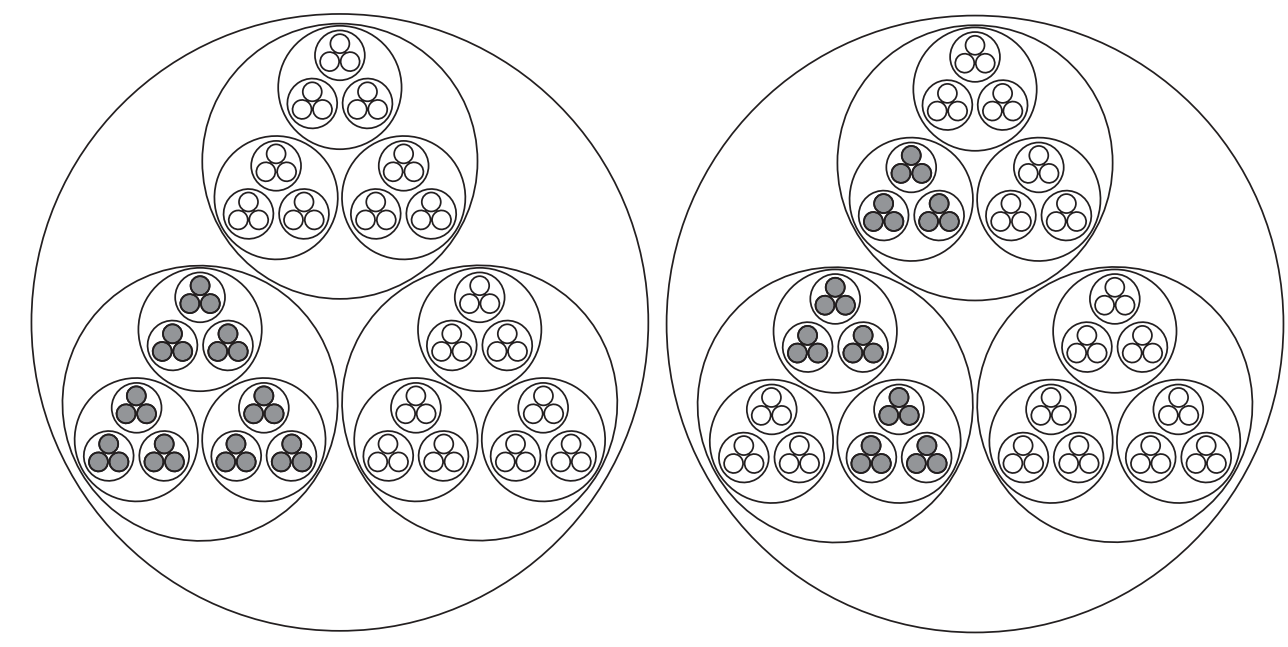

FIGURE $7 \quad \Omega_{1,0}$ and $\Omega_{1,1}$ of Example 5.3. 


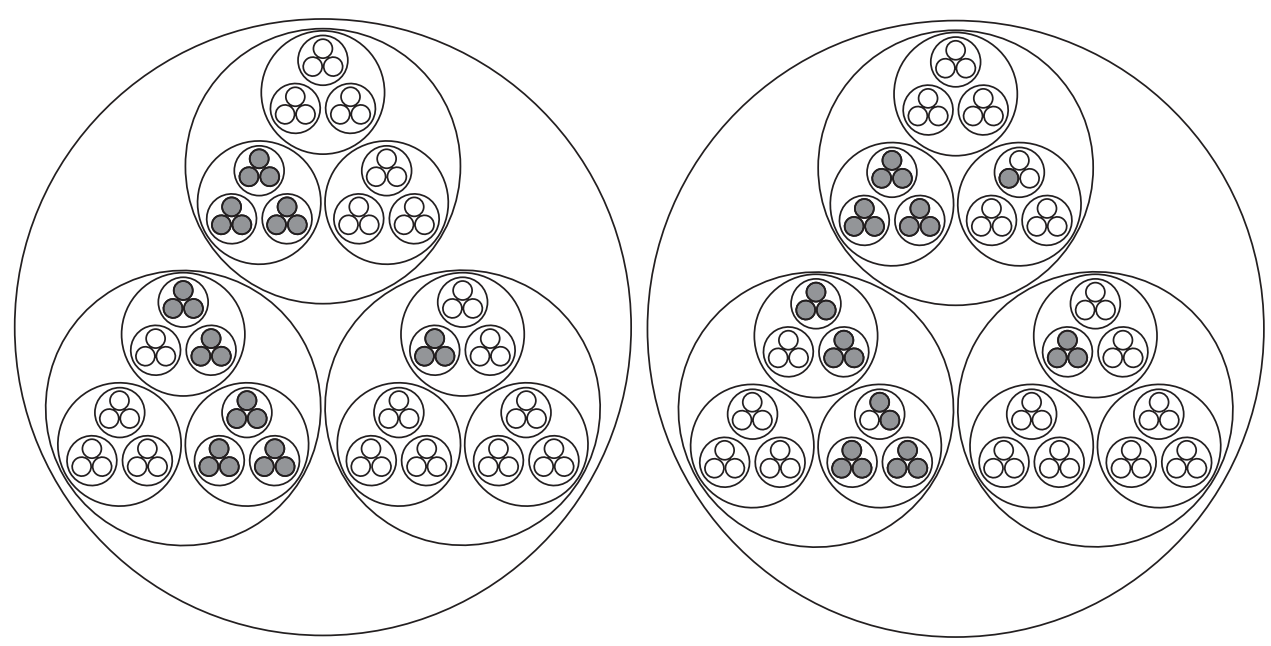

FIGURE $8 \Omega_{1,2}$ and $\Omega_{1,3}$ of Example 5.3.

Thus, $\Lambda_{1}=\bigcup_{m \geq 1} \Lambda_{1, m} \subseteq \mathbb{Z}_{3}$ is a disjoint union of countably many disks, with $v\left(\Lambda_{1}\right)=$ $1 /(3-1)=1 / 2$, and the wavelet set is

$$
\begin{aligned}
\Omega_{1}=\left[\mathbb{Z}_{3} \backslash \bigcup_{n=1}^{\infty}\left(\left(-5 / 8+3^{2 n-2}+3^{2 n-1} \mathbb{Z}_{3}\right) \cup\left(-7 / 8+3^{2 n-1}+3^{2 n} \mathbb{Z}_{3}\right)\right)\right] \\
\cup \bigcup_{m=1}^{\infty}\left(\left(-7 / 24+3^{2 n-2}+3^{2 n-1} \mathbb{Z}_{3}\right) \cup\left(-5 / 24+3^{2 n-1}+3^{2 n} \mathbb{Z}_{3}\right)\right) .
\end{aligned}
$$

\section{Acknowledgments}

Many of the references reflect fruitful conversations about our program that the authors had with both analysts and number theorists. In particular, and not necessarily referenced, we want to thank Professors Lawrence Baggett, David Cox, Hans Feichtinger, Karlheinz Gröchenig, and Niranjan Ramachandran.

\section{References}

[1] Ali, S.T., Antoine, J.-P., and Gazeau, J.P-. Coherent States, Wavelets and their Generalizations, Springer-Verlag, New York, (2000).

[2] Altaiski, M.V. p-adic wavelet decomposition vs Fourier analysis on spheres, Indian J. Pure Appl. Math., 28(2), 197-205, (1997).

[3] Antoine, J.-P., Kouagou, Y.B., Lambert, D., and Torrésani, B. An algebraic approach to discrete dilations. Application to discrete wavelet transforms, J. Fourier Anal. Appl., 6(2), 113-141, (2000).

[4] Antoine, J.-P. and Vandergheynst, P. Wavelets on the 2-sphere: a group-theoretical approach, Appl. Comput. Harmon. Anal., 7(3), 262-291, (1999).

[5] Baggett, L.W., Medina, H.A., and Merrill, K.D. Generalized multi-resolution analyses and a construction procedure for all wavelet sets in $\mathbf{R}^{n}$, J. Fourier Anal. Appl., 5(6), 563-573, (1999).

[6] Baggett, L.W. and Merrill, K.D. Abstract harmonic analysis and wavelets in $\mathbf{R}^{n}$, The Functional and Harmonic Analysis of Wavelets and Frames, (San Antonio, TX, 1999), Am. Math. Soc., 17-27, Providence, RI, (1999).

[7] Benedetto, J.J. Spectral Synthesis, Academic Press, New York, (1975).

[8] Benedetto, J.J. Frames, sampling, and seizure prediction, in Advances in Wavelets, Lau, K.-S., Ed., Springer-Verlag, New York, (1998).

[9] Benedetto, J.J. and Benedetto, R.L. Multiresolution analysis over local fields, in preparation, (2004). 
[10] Benedetto, J.J. and Leon, M.T. The construction of multiple dyadic minimally supported frequency wavelets on $\mathbb{R}^{d}$, Contemp. Math., 247, 43-74, (1999).

[11] Benedetto, J.J. and Leon, M.T. The construction of single wavelets in $d$-dimensions, J. Geom. Anal., 11(1), 1-15, (2001).

[12] Benedetto, J.J. and Sumetkijakan, S. A fractal set constructed from a class of wavelet sets, Contemp. Math., 313, 19-35, (2002).

[13] Benedetto, J.J. and Sumetkijakan, S. Tight frames and geometric properties of wavelet sets, Adv. Comp. Math., (2004).

[14] Benedetto, R.L. Examples of wavelets for local fields, Wavelets, Frames, and Operator Theory, (College Park, MD, 2003), Am. Math. Soc., 27-47, Providence, RI, (2004).

[15] Casazza, P.G., Han, D., and Larson, D.R. Frames for Banach spaces, The Functional and Harmonic Analysis of Wavelets and Frames, (San Antonio, TX, 1999), Am. Math. Soc., 149-182, Providence, RI, (1999).

[16] Courter, J. Construction of dilation- $d$ wavelets, AMS Contemp. Math., 247, 183-205, (1999).

[17] Dahlke, S. Multiresolution analysis and wavelets on locally compact abelian groups, in Wavelets, Images, and Surface Fitting, (Chamonix-Mont-Blanc, 1993), Peters, A.K., Wellesley, MA, 141-156, (1994).

[18] Dahlke, S. The construction of wavelets on groups and manifolds, in General Algebra and Discrete Mathematics, (Potsdam, 1993), Heldermann, Lemgo, 47-58, (1995).

[19] Dai, X. and Larson, D.R. Wandering vectors for unitary systems and orthogonal wavelets, Mem. Am. Math. Soc., 134(640), viii+68, (1998).

[20] Dai, X., Larson, D.R., and Speegle, D.M. Wavelet sets in $\mathbf{R}^{n}$, J. Fourier Anal. Appl., 3(4), 451-456, (1997).

[21] Dai, X., Larson, D.R., and Speegle, D.M. Wavelet sets in $\mathbf{R}^{n}$, II, Wavelets, Multiwavelets, and Their Applications, (San Diego, CA, 1997), Am. Math. Soc., Providence, RI, 15-40, (1998).

[22] Daly, J. and Phillips, K. On singular integrals, multipliers, $\mathfrak{H}^{1}$ and Fourier series-a local field phenomenon, Math. Ann., 265(2), 181-219, (1983).

[23] Daubechies, I. Ten Lectures on Wavelets, CBMS-NSF Series in Applied Math., SIAM, Philadelphia, PA, (1992).

[24] Farkov, Yu.A. Orthogonal wavelets on locally compact abelian groups, Funktsional. Anal. i Prilozhen., 31(4), 86-88, (1997).

[25] Flornes, K., Grossmann, A., Holschneider, M., and Torrésani, B. Wavelets on discrete fields, Appl. Comput. Harmon. Anal., 1(2), 137-146, (1994).

[26] Gouvêa, F. p-adic Numbers, an Introduction, 2nd ed., Springer-Verlag, Berlin, (1997).

[27] Gröchenig, K. and Haas, A. Self-similar lattice tilings, J. Fourier Anal. Appl., 1(2), 131-170, (1994).

[28] Gröchenig, K. and Madych, W.R. Multiresolution analysis, Haar bases, and self-similar tilings of $L^{2}\left(\mathbf{R}^{n}\right)$, IEEE Trans. Inform. Th., 38(2), 556-568, (1992).

[29] Han, D., Larson, D.R., Papadakis, M., and Stavropoulos, T. Multiresolution analyses of abstract Hilbert spaces and wandering subspaces, The Functional and Harmonic Analysis of Wavelets and Frames, (San Antonio, TX, 1999), Am. Math. Soc., Providence, RI, 259-284, (1999).

[30] Hernández, E., Wang, X., and Weiss, G. Smoothing minimally supported frequency wavelets, part II, J. Fourier Anal. Appl., 3(1), 23-41, (1997).

[31] Hewitt, E. and Ross, K.A. Abstract Harmonic Analysis, Volume I, Springer-Verlag, New York, (1963).

[32] Hewitt, E. and Ross, K.A. Abstract Harmonic Analysis, Volume II, Springer-Verlag, New York, (1970).

[33] Holschneider, M. Wavelet analysis over abelian groups, Appl. Comput. Harmon. Anal., 2(1), 52-60, (1995).

[34] Johnston, C.P. On the pseudodilation representations of Flornes, Grossmann, Holschneider, and Torresani, $J$. Fourier Anal. Appl., 3(4), 377-385, (1997).

[35] Koblitz, N. p-adic Numbers, p-adic Analysis, and Zeta-Functions, 2nd ed., Springer-Verlag, New York, (1984).

[36] Kozyrev, S.V. Wavelet analysis as a $p$-adic spectral analysis, Izv. Math., 66, 367-376, (2002).

[37] Lagarias, J.C. and Wang, Y. Haar type orthonormal wavelet bases in $\mathbf{R}^{2}$, J. Fourier Anal. Appl., 2(1), 1-14, (1995).

[38] Lagarias, J.C. and Wang, Y. Integral self-affine tiles in $\mathbf{R}^{n}$, part II lattice tilings, J. Fourier Anal. Appl., 3(1), 83-102, (1997).

[39] Lang, W.C. Orthogonal wavelets on the Cantor dyadic group, SIAM J. Math. Anal., 27, 305-312, (1996),.

[40] Lang, W.C. Fractal multiwavelets related to the Cantor dyadic group, Internat. J. Math. Math. Sci., 21, 307-314, (1998).

[41] Lang, W.C. Wavelet analysis on the Cantor dyadic group, Houston J. Math., 24, 533-544 and 757-758, (1998).

[42] Lemarié, P.G. Base d'ondelettes sur les groupes de Lie stratifiés, Bull. Soc. Math. France, 117(2), 211-232, (1989).

[43] Lukashenko, T.P. Wavelets on topological groups, Izv. Ross. Akad. Nauk. Ser. Mat., 58(3), 88-102, (1994). 
[44] Madych, W.R. Some elementary properties of multiresolution analyses of $L^{2}\left(\mathbf{R}^{n}\right)$, in Wavelets: a Tutorial in Theory and Applications, Chui, C.K., Ed., Academic Press, San Diego, CA, 256-294, (1992).

[45] Mallat, S. A Wavelet Tour of Signal Processing, Academic Press, Boston, (1998).

[46] Meyer, Y. Ondelettes et Opérateurs, Hermann, Paris, (1990).

[47] Papadakis, M. Generalized frame multiresolution analysis of abstract Hilbert spaces, Sampling, Wavelets, and Tomography, Birkhäuser, Boston, MA, (2003).

[48] Papadakis, M. and Stavropoulos, T. On the multiresolution analyses of abstract Hilbert spaces, Bull. Greek Math. Soc., 40, 79-92, (1998).

[49] Pontryagin, L.L. Topological Groups, 2nd ed., Gordon and Breach, Science Publishers, New York, (1966), transl. from the Russian by Arlen Brown.

[50] Ramakrishnan, D. and Valenza, R.J. Fourier Analysis on Number Fields, Springer-Verlag, New York, (1999).

[51] Reiter, H. Classical Harmonic Analysis and Locally Compact Groups, Oxford University Press, (1968).

[52] Robert, A.M. A Course in p-adic Analysis, Springer-Verlag, New York, (2000).

[53] Rudin, W. Fourier Analysis on Groups, John Wiley \& Sons, New York, (1962).

[54] Schulz, E. and Taylor, K.F. Extensions of the Heisenberg group and wavelet analysis in the plane, Spline Functions and the Theory of Wavelets, (Montreal, PQ, 1996), Am. Math. Soc., Providence, RI, 217-225, (1999),

[55] Serre, J.-P. Local Fields, Springer-Verlag, New York, (1979), transl. by Marvin J. Greenberg.

[56] Soardi, P.M. and Weiland, D. Single wavelets in $n$-dimensions, J. Fourier Anal. Appl., 4(3), 299-315, (1998).

[57] Taibleson, M.H. Fourier Analysis on Local Fields, Princeton University Press, Princeton, NJ, (1975).

[58] Trimèche, K. Continuous wavelet transform on semisimple Lie groups and inversion of the Abel transform and its dual, Collect. Math., 47(3), 231-268, (1996).

[59] Zakharov, V.G. Nonseparable multidimensional Littlewood-Paley like wavelet bases, preprint, (1996).

Received May 25, 2004

Department of Mathematics, University of Maryland, College Park, MD 20742

e-mail: jjb@math.umd.edu

http: / / www . math. umd.edu/ jjb

Department of Mathematics and Computer Science, Amherst College, Amherst, MA 01002-5000

e-mail: rlb@cs.amherst.edu

http://www.cs.amherst.edu/ rlb

Communicated by Guido Weiss 